JOURNAL OF THE

AMERICAN MATHEMATICAL SOCIETY

Volume 14, Number 1, Pages 239-262

S 0894-0347(00)00354-4

Article electronically published on September 25, 2000

\title{
DOUBLE AFFINE HECKE ALGEBRAS AND 2-DIMENSIONAL LOCAL FIELDS
}

\author{
M. KAPRANOV
}

The concept of an $n$-dimensional local field was introduced by A.N. Parshin [Pa1] with the aim of generalizing the classical adelic formalism to (absolutely) $n$ dimensional schemes. By definition, a 0-dimensional local field is just a finite field, and an $n$-dimensional local field, $n>0$, is a complete discrete valued field whose residue field is $(n-1)$-dimensional local. Thus for $n=1$ we get locally compact fields such as $\mathbf{Q}_{p}, F_{q}((t))$, and for $n=2$ we get fields such as $\mathbf{Q}_{p}((t)), F_{q}\left(\left(t_{1}\right)\right)\left(\left(t_{2}\right)\right)$, etc.

In representation theory, harmonic analysis on reductive groups over 0- and 1dimensional local fields leads, in particular, to consideration of the finite and affine Hecke algebras $H_{q}, \dot{H}_{q}$ associated to any finite root system $R$ and any $q \in \mathbf{C}^{*}$. These algebras can be defined in several ways, one being by generators and relations, another as the convolution algebra, with respect to the Haar measure, of functions on the group bi-invariant with respect to an appropriate subgroup (i.e., as the algebra of double cosets). Harmonic analysis on groups over 2-dimensional local fields has not been developed, the main difficulty being the infinite dimensionality (absense of local compactness) of such fields. However, the double affine Hecke algebra $\ddot{H}_{q}$ recently defined by I. Cherednik Ch in terms of generators and relations, looks like the third term in the hierarchy starting from $H_{q}, \dot{H}_{q}$. The problem "give a group-theoretic construction of the Cherednik algebra" (i.e., realize it as some algebra of double cosets) was proposed by D. Kazhdan a few years ago.

The purpose of the present paper is to provide a solution to this problem by developing beginnings of harmonic analysis on reductive groups over 2-dimensional local fields. We consider a simple algebraic group $G$ (over $\mathbf{Z}$ ), a 2-dimensional local field $K=k((t))$ of equal characteristic (so $k$ is 1-dimensional local) and the canonical central extension $\Gamma$ of $G(K)$ by $k^{*}$. For an appropriate subgroup $\Delta_{1} \subset \Gamma$ the fibers of the Hecke correspondences (3.1) are locally compact spaces (affine spaces over $k$ of growing dimension) which possess natural invariant measures, so one can formally define the Hecke operators associated to double cosets by integrating over these measures. The main difficulty here is the noncompactness of the domain of integration. It poses convergence problems, making it unclear how to compose such operators or how to define their action from some vector space to itself. More precisely, the operators are well defined on the space $\mathcal{F}_{0}$ of functions on $\Gamma / \Delta_{1}$ with certain proper support conditions but their values lie in a bigger space $\mathcal{F}$.

Received by the editors June 8, 1999 and, in revised form, March 16, 2000 and July 25, 2000. 2000 Mathematics Subject Classification. Primary 20C08; Secondary 20 G25. 
The way around the difficulty that we take is to use the analytic continuation with respect to the parameters of the principal series representations. This is a version of the classical method of regularizing divergent integrals by introducing complex powers of auxiliary polynomials into the integrand, so that the regularized integral comes out as a function (possibly meromorphic) of the complex exponents. In our nonarchimedean case such functions are rational, in appropriate variables. Then, we define the regularized Hecke algebra $H\left(\Gamma, \Delta_{1}\right)$ to consist of certain linear combinations of the regularized Hecke operators with coefficients being rational functions of the principal series exponents. The rule to single out the admissible linear combinations is that they should preserve the space $\mathcal{F}_{0}$. The main result (Theorem 3.3.8) is that $H\left(\Gamma, \Delta_{1}\right)$ is isomorphic to the (slight modification of) the Cherednik algebra associated to $G$.

These analytic difficulties seem unavoidable because we are dealing with an infinite-dimensional group with no Haar measure. The approach presented here is not restricted to our particular choice of the subgroup $\Delta_{1}$ but can be applied to other choices as well, in particular, to arbitrarily "deep" congruence subgroups $\Delta^{\prime} \subset \Delta_{1}$ (with $\Delta_{1} / \Delta^{\prime}$ locally compact). As in the familiar p-adic case, the resulting algebras, while not possessing a nice independent description, are nevertheless important for the general theory of representations of infinite-dimensional groups such as $\Gamma$.

Another general point which seems important for the future is the essential role of iterated ind- and pro-objects in the representation theory of groups over 2dimensional local fields. Thus, the "spaces" of the principal series representations are in fact pro-vector spaces, the group itself is not a topological group at all but rather a group object in a certain iterated pro-ind-category and so on. The philosophy that topological concepts when applied to $n$-dimensional local fields, $n \geq 2$, become inadequate and should be replaced by considering ind/pro objects was explicitly formulated by K. Kato Kat. In the present paper we use ind/proobjects in a systematic way.

In order to keep the paper short, it is organized as follows. Section 1 contains the setup for the groups and homogeneous spaces we consider, including the issues related to the central extension. This material is mostly well known. Section 2 is a reminder of the Cherednik algebras. In Section 3 we explain our approach to constructing the regularized Hecke algebra and formulate the main theorem. We tried to split the construction into several steps so that they are easily generalizable to more complicated situations. Section 4 contains the proof of the main theorem by using the principal series intertwiners and a version of the Mellin transform. Finally, the Appendix contains the general constructions on ind/pro objects (such as function spaces, etc.) necessary for the main text. Its content is used throughout the paper.

Among the (already fairly numerous) works on the Cherednik algebras two are most relevant for this paper. One is the paper [GG] which gives a construction in terms of equivariant K-theory, generalizing the results of $[\mathrm{KL}$. The other one is GKV] where an "elementary" description was given by unraveling [KL, GG] in terms of certain residue conditions. This description is in fact used here in an essential way, as the conditions of [GKV] match very precisely the singularity properties of the principal series intertwiners.

I would like to thank A. Braverman, J. Bernstein, S. Evens, V. Ginzburg and A. Parshin for useful discussions and correspondence. In particular, work with 
V. Ginzburg on a related project stimulated my thinking about the questions addressed here. The work on this paper was partially supported by an NSF grant and a large part of it was carried out during a visit to the IHES in the summer of 1998 .

\section{§1. P-adic loop groups and their homogeneous spaces}

(1.1) Root systems. We start by introducing notation to be used in the rest of the paper. Let $G$ be a split simple, simply-connected algebraic group (over $\mathbf{Z}$ ), $T \subset B \subset G$ the fixed maximal torus and Borel subgroup, $N=[B, B]$. We regard $G, B, N, T$ as group schemes.

Let $L=\operatorname{Hom}\left(\mathbf{G}_{m}, T\right)$ and $L^{\vee}=\operatorname{Hom}\left(T, \mathbf{G}_{m}\right)$ be the coweight and weight lattices of $G ; R \subset L^{\vee}$ the root system, $R_{\text {sim }} \subset R_{+} \subset R$ the sets of simple and positive roots. For $\alpha \in R_{+}$let $\alpha^{\vee} \in L$ be the corresponding coroot. Let also $\theta \in R_{+}$be the maximal root and $\rho \in L^{\vee}$ the half-sum of positive roots. By $h^{\vee}$ we denote the dual Coxeter number of $G$.

We denote by $\check{T}=$ Spec $\mathbf{C}[L]$ the complex torus dual to $T$. Let $W$ be the Weyl group of $G$. It acts on $L$ and $L^{\vee}$. Denote by

$$
\Psi: L \rightarrow L^{\vee}, \quad \Psi(a)=\frac{1}{h^{\vee}} \sum_{\alpha \in R_{+}}(\alpha, a) \alpha
$$

the minimal $W$-invariant integral scalar product on $L$; see $[\mathrm{Kac}, \S 6$.

Let $L_{\text {aff }}=\mathbf{Z} \oplus L, L_{\text {aff }}^{\vee}=\mathbf{Z} \oplus L^{\vee}$ be the lattices of affine (co)weights of $G$. They are dual to each other. Let

$$
\widehat{R}=\{(n, \alpha), \alpha \in R, n \in \mathbf{Z}\} \subset L_{\text {aff }}^{\vee}, \quad \widehat{R}_{+}=\left(\{0\} \times R_{+}\right) \cup\left(\mathbf{Z}_{>0} \times R\right)
$$

be the system of affine roots of $G$ and the set of positive affine roots. The set of simple affine roots will be denoted by

$$
\widehat{R}_{\text {sim }}=\left(\{0\} \times R_{\text {sim }}\right) \cup\{(1,-\theta)\} .
$$

Let $\widehat{W}=W \propto L$ be the affine Weyl group of $G$, generated by the reflections $s_{\alpha}, \alpha \in \widehat{R}_{+}$. We denote by $\leq$the Bruhat order on $\widehat{W}$. Let $l: \widehat{W} \rightarrow \mathbf{Z}_{+}$be the length function. The group $\widehat{\widehat{W}}$ acts $\mathbf{Z}$-linearly on $L_{\text {aff }}^{\vee}$ by

$$
w(m, b)=(m, w(b)), w \in W, \quad a(m, b)=(m+(a, b), b), a \in L .
$$

We will also need the action on $L_{\text {aff }}$ given by

$$
w \circ(m, b)=(m, w(b)), w \in W, \quad a \circ(m, b)=(m+\Psi(a, b), b), a \in L .
$$

The action (1.1.2) preserves $\widehat{R}$, and we set

$$
D(w)=\left\{\alpha \in \widehat{R}_{+}: w(\alpha) \in \widehat{R}_{-}\right\}, \quad w \in \widehat{W} .
$$

Then $|D(w)|=l(w)$. Let

$$
\widehat{\rho}=\left(-h^{\vee}, \rho\right) \in L_{\text {aff }}^{\vee}
$$

be the standard substitute for the half-sum of positive affine roots; see [PS], $\S 14.3$. It satisfies $\left(\widehat{\rho}, \alpha^{\vee}\right)=1$ for any $\alpha \in \widehat{R}_{\text {sim }}$. We set

$$
\widehat{\delta}(w)=\widehat{\rho}-w(\widehat{\rho})=\sum_{\alpha \in D(w)} \alpha, \quad w \in \widehat{W} .
$$


Let also

$$
T_{\text {aff }}(\mathbf{C})=\operatorname{Spec} \mathbf{C}\left[L_{\text {aff }}^{\vee}\right], \quad \check{T}_{\text {aff }}=\operatorname{Spec} \mathbf{C}\left[L_{\text {aff }}\right]
$$

be the affine tori corresponding to $T(\mathbf{C}), \check{T}$.

(1.2) Groups and homogeneous spaces. Let $k$ be a complete discrete valued field with residue field $F_{q}$ and $K=k((t))$. So $K$ is a complete discrete valued field with residue field $k$, thus a 2-dimensional local field in the sense of Pa1. We use the notations $\mathcal{O}_{K}=k[[t]]$ and $\mathbf{m}_{K}=t k[[t]]$ for the ring of integers and maximal ideal of $K$, and $\mathcal{O}_{k}$ and $\mathbf{m}_{k}$ for the analogous subrings in $k$. Denote by $\pi_{K}: \mathcal{O}_{K} \rightarrow k$, $\pi_{k}: \mathcal{O}_{k} \rightarrow F_{q}$ the projections and set $\mathcal{O}^{\prime}=\pi_{K}^{-1}\left(\mathcal{O}_{k}\right) \subset \mathcal{O}_{K}$. The quotient $K^{*} / \mathcal{O}^{*}$ will be denoted $\epsilon$. It is a free Abelian group of rank 2 fitting into a natural exact sequence

$$
0 \rightarrow \mathbf{Z} \rightarrow \epsilon \rightarrow \mathbf{Z} \rightarrow 0
$$

Let $G$ be as in (1.1). The semidirect product $\widehat{\widehat{W}}=W \propto(L \otimes \epsilon)$ will be called the double affine Weyl group for $G$; see $\mathrm{Pa} 2$. Consider the group $G(K)$ and the following three subgroups:

$$
\begin{aligned}
& D_{0}=\left\{g \in G\left(\mathcal{O}^{\prime}\right): \pi_{k}\left(\pi_{K}(g)\right) \in B\left(F_{q}\right)\right\}, \\
& D_{1}=\left\{g \in G\left(\mathcal{O}_{K}\right): \pi_{K}(g) \in T\left(\mathcal{O}_{k}\right) N(k)\right\}, \\
& D_{2}=T\left(\mathcal{O}^{\prime}\right) N(K) .
\end{aligned}
$$

Thus $D_{2}$ is a natural "connected component" of the Borel subgroup in $G(K), D_{1}$ is a similar connected component of the Iwahori subgroup in $G(K)$ (where $K$ is considered just as a local field with residue field $k$ ), and $D_{0}$ is the "double-Iwahori" subgroup (cf. $\mathrm{Pa} 2$ ).

Let $\varpi_{1} \in \mathbf{m}_{k}$ be a uniformizer of $k$ and $\varpi_{2}=t$ the standard uniformizer of $K$. This choice of uniformizers gives an identification

$$
\mathbf{Z}^{2} \rightarrow \epsilon, \quad(i, j) \mapsto \varpi_{1}^{i} \varpi_{2}^{j} \bmod \left(\mathcal{O}^{\prime}\right)^{*}
$$

and therefore an identification $L \oplus L \rightarrow L \otimes \epsilon$, as well as a realization of $L \otimes \epsilon$ as a subgroup in $K^{*}$.

(1.2.3) Proposition. For any $i, j=0,1,2$ we have the decomposition

$$
G(K)=\coprod_{w \in \widehat{\widehat{W}}} D_{i} w D_{j}
$$

and the resulting identification $D_{i} \backslash \Gamma / D_{j} \rightarrow \widehat{\widehat{W}}$ is canonical (independent of the choice of liftings of elements of $W$ to elements of $G(K))$.

Proof. If $E$ is a field, we have the Bruhat decomposition $G(E)=\coprod_{w \in W} B(E) w B(E)$. If $(E, \bar{E})$ is a local field, then we have the Bruhat-Tits and Iwasawa decompositions

$$
G(E)=\coprod_{w \in \widehat{W}} I w I=\coprod_{a \in L} G\left(\mathcal{O}_{E}\right) a N(E)
$$

where $I$ is the Iwahori subgroup. The proposition is obtained by iterated application of the Bruhat decomposition to the fields $K, k, F_{q}$ and of the Bruhat-Tits and Iwasawa decompositions to the local fields $(K, k)$ and $\left(k, F_{q}\right)$; see the argument in Pa2, $§ 2$, for the group $P G L_{n}$. 
In this paper we will be mostly interested in the subgroup $D_{1}$.

Let $I=\pi_{K}^{-1}(B(k))$ be the Iwahori subgroup in $G(K)$. Consider the following homogeneous spaces:

$$
\widehat{X}=G(K) / G\left(\mathcal{O}_{K}\right), \quad \widehat{F}=G(K) / I_{K}, \quad \mathcal{M}=G(K) / D_{1} .
$$

The set $\widehat{F}$ is the "affine flag variety" of $G$, and $\widehat{X}$ is the "affine Grassmannian"; see Lu1. We have the projections

$$
p_{1}: \widehat{F} \rightarrow \widehat{X}, \quad p_{2}: \mathcal{M} \rightarrow \widehat{F}
$$

with fibers of $p_{1}$ isomorphic to $F=G(k) / B(k)$ and the fibers of $p_{2}$ being $L$-torsors.

The Bruhat-Tits decomposition associated to $G$ and the local field $(K, k)$ allows us to speak about the relative position $w\left(b, b^{\prime}\right) \in \widehat{W}$ of two points $b, b^{\prime} \in \widehat{F}$. Denote by $U_{w}(b) \subset \widehat{F}$ the set of $b^{\prime}$ such that $w\left(b, b^{\prime}\right)=w$ (affine Schubert cell). Set also

$$
\bar{U}_{w}(b)=\bigcup_{w^{\prime} \leq w} U_{w}(b),
$$

where $w^{\prime} \leq w$ stands for the Bruhat order. For the following, see [Lu1].

(1.2.7) Theorem. Each $\bar{U}_{w}(b)$ has a natural structure of a projective algebraic variety over $k$ of dimension $l(w)$, so that $U_{w}(b)$ is an open subvariety isomorphic to the affine space. The set $\widehat{F}$ has therefore a structure of (the set of $k$-points of) an ind-object in the category of projective algebraic varieties over $k$. The group $G(K)$ acts on $\widehat{F}$ by automorphisms of an ind-object.

(1.2.8) Corollary. $\widehat{F}$ has a natural topology, the inductive limit of compact totally disconnected topologies on the $\bar{U}_{w}(b)$. The action of $G(K)$ is by homeomorphisms.

(1.3) The central extension. Recall the general theory of Steinberg-MooreMatsumoto Mat. Let $E$ be any field, $B$ an Abelian group and $s: E^{*} \times E^{*} \rightarrow B$ a Steinberg symbol, i.e., a bi-homomorphic map satisfying the identity $s(x, 1-x)=1$. To this data Matsumoto associates a central extension

$$
1 \rightarrow B \rightarrow \widetilde{G}(E)_{s} \stackrel{p}{\rightarrow} G(E) \rightarrow 1 .
$$

As with any central extension, the extension induced by (1.3.1) on $T(E)$ gives rise to the commutator map

$$
c_{s}: T(E) \times T(E) \rightarrow B, \quad c_{s}(x, y)=[\widetilde{x}, \widetilde{y}] \in B, p(\widetilde{x})=x, p(\widetilde{y})=y,
$$

which is characterized by the property:

$$
c_{s}\left(\lambda^{a}, \mu^{b}\right)=c(\lambda, \mu)^{\Psi(a, b)}, \quad \lambda, \mu \in K^{*}, a, b \in L=\operatorname{Hom}\left(\mathbf{G}_{m}, T\right) .
$$

Here $\Psi$ is as in (1.1.1). We specialize this construction to $E=K, B=k^{*}$ and $s$ being the tame symbol

$$
s_{\text {tame }}(x, y)=(-1)^{\operatorname{ord}(x) \operatorname{ord}(y)} \pi\left(\frac{x^{\operatorname{ord}(y)}}{y^{\operatorname{ord}(x)}}\right) .
$$

We denote the corresponding central extension by

$$
1 \rightarrow k^{*} \rightarrow \Gamma \rightarrow G(K) \rightarrow 1
$$

(1.3.6) Proposition. The extension $\Gamma$ canonically splits over any of the subgroups $D_{i} \subset \Gamma$ defined in (1.2.2). 
Proof. Matsumoto's explicit construction of $\widetilde{G}(E)_{s}$ for any $E, s$ is done in two steps; see Mat, Mil. First, one considers the group scheme $M=\operatorname{Norm}(T)$, the normalizer of $T$, and constructs a central extension $\widetilde{M}(E)_{s} \rightarrow M(E)$ by using $s$. Then, one considers the retraction $\rho: G(E) \rightarrow M(E)$ given by the Bruhat decomposition, i.e., $\rho(g)$ is the unique element $m \in M(E)$ such that $g=u_{1} m u_{2}$ with $u_{i} \in N(E)$. The extension $\widetilde{G}(E)_{s}$ is defined as a subgroup of bijections of $\widetilde{M}(E) \times_{M(E)} G(E)$ generated by certain bijections $u_{\alpha}(e), e \in E, h_{\alpha}(e), e \in E^{*}, w_{\alpha}$, $\alpha \in R_{+}$, mimicking respectively the action of Chevalley generators from $N(E)$, of the elements of $T(E)$ and of elements of $W$ labelled by $\alpha$. In our case $E=K, B=$ $k^{*}, s=s_{\text {tame }}$. So it is clear that the extension is trivial on $D_{2}=N(E) T\left(\mathcal{O}^{\prime}\right)$, as $s$ vanishes on $\mathcal{O}^{*} \supset\left(\mathcal{O}^{\prime}\right)^{*}$. As for $D_{i}, i=0,1$, they both lie in $G(\mathcal{O})$, and in fact the extension is trivial over $G(\mathcal{O})$ because of the decomposition $G(\mathcal{O})=$ $N(\mathcal{O}) M(\mathcal{O}) N(\mathcal{O})$ and again of triviality of the tame symbol on $\mathcal{O}^{*}$.

According to the above proposition, we can and will view $D_{i}$ as subgroups in $\widetilde{\Gamma}$ and denote $\Delta_{i}=\mathcal{O}_{k}^{*} \cdot D_{i} \subset \Gamma$. We will be mostly interested in the case $i=1$ and denote $\Xi=\Gamma / \Delta_{1}$. Thus $\Xi \rightarrow \mathcal{M}=\Gamma / \Delta_{1}$ is a $\mathbf{Z}$-torsor, or, equivalently,

$$
p: \Xi \rightarrow \widehat{F}
$$

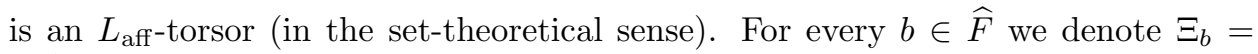
$p^{-1}(b)$.

(1.4) The affine Heisenberg-Weyl group. Note that the composite map

$$
K^{*} \otimes \mathbf{Z} K^{*} \stackrel{\{,\}}{\longrightarrow} k^{*} \stackrel{\operatorname{ord}_{k}}{\longrightarrow} \mathbf{Z}
$$

descends to a nondegenerate skew-symmetric pairing $\sigma: \Lambda^{2}(\epsilon) \rightarrow \mathbf{Z}$, where $\epsilon$ is as in (1.2.1). We get therefore a $W$-invariant skew-symmetric pairing $\Psi \otimes \sigma$ on $L \otimes \epsilon$ and can form the corresponding central extension

$$
1 \rightarrow \mathbf{Z} \rightarrow \widetilde{L \otimes \epsilon} \rightarrow L \otimes \epsilon \rightarrow 1
$$

(the Heisenberg group) for which the commutator pairing is equal to $\Psi \otimes \sigma$. This pairing being $W$-invariant, we have a natural $W$-action on $\widetilde{L \otimes \epsilon}$.

(1.4.2) Definition. The (double) affine Heisenberg-Weyl group is the semidirect product $\widetilde{W}:=W \propto(\widetilde{L \otimes \epsilon})$.

The following is then straightforward.

(1.4.3) Proposition. $\widetilde{W}$ is isomorphic to $\widehat{W} \propto L_{\text {aff }}$, the semidirect product with respect to the action (1.1.3).

Proposition (1.2.3) implies easily:

(1.4.4) Proposition. For any $i, j=0,1,2$ we have the decomposition

$$
\Gamma=\coprod_{w \in \widetilde{W}} \Delta_{i} w \Delta_{j} .
$$

From this statement we deduce the following. 
(1.4.6) Proposition. (a) For every $b, b^{\prime} \in \widehat{F}$ such that $w\left(b, b^{\prime}\right)=w \in \widehat{W}$, we have a natural identification of torsors $j_{b b^{\prime}}: \Xi_{b} \rightarrow \Xi_{b^{\prime}}$, compatible with the identification $w: L_{\mathrm{aff}} \rightarrow L_{\mathrm{aff}}$ of the structure groups. These identifications are $\Gamma$-equivariant, i.e., we have

$$
g\left(j_{b b^{\prime}}(\xi)\right)=j_{g(b), g\left(b^{\prime}\right)}(g(\xi)), \quad g \in \Gamma,
$$

and satisfy the transitivity conditions: $j_{b b^{\prime \prime}}=j_{b^{\prime} b^{\prime \prime}} \circ j_{b b^{\prime}}$ for any $b, b^{\prime}, b^{\prime \prime} \in \widehat{F}$.

(b) The $\Gamma$-orbit on $\Xi \times \Xi$ associated to $(w, l) \in \widetilde{W}=\widehat{W} \propto L_{\text {aff }}$ is the subset

$$
\Sigma_{w, l}=\left\{\left(\xi, \xi^{\prime}\right) \in \Xi \times \Xi \mid \xi \in \Xi_{b}, \xi^{\prime} \in \Xi_{b^{\prime}}, w\left(b, b^{\prime}\right)=w, \xi^{\prime}=j_{b b^{\prime}}(\xi)+l\right\} .
$$

In particular, $p: \Xi \rightarrow \widehat{F}$ identifies $\Sigma_{w, l} \cap(\{\xi\} \times \Xi)$ with the Schubert cell $U_{w}(p(\xi))$.

Proof. (a) Let $N=\left[\Delta_{1}, \Delta_{1}\right]$, so $\Delta_{1} / N=T^{\text {aff }}(k)$. Note that $L_{\text {aff }}=T^{\text {aff }}(k) / T^{\text {aff }}\left(\mathcal{O}_{k}\right)$. Consider the projection

$$
\pi: \Gamma / N \rightarrow \Gamma / \Delta_{1}=\widehat{F}
$$

and denote its fibers by $\mathcal{T}_{b}=\pi^{-1}(b)$. They are $T^{\text {aff }}(k)$-torsors, and $\Xi_{b}=\mathcal{T}_{b} / T^{\text {aff }}\left(\mathcal{O}_{k}\right)$. So we will construct an identification $J_{b b^{\prime}}: \mathcal{T}_{b} \rightarrow \mathcal{T}_{b^{\prime}}$ of $T^{\text {aff }}(k)$-torsors, compatible with the identification of structure groups $w: T^{\text {aff }}(k) \rightarrow T^{\text {aff }}(k)$, and define $j_{b b^{\prime}}$ to be the map induced by $J_{b b^{\prime}}$ on the quotients by $T^{\text {aff }}\left(\mathcal{O}_{k}\right)$. For this, consider an element $x \in \mathcal{T}_{b}$. As $x$ is an element of $\Gamma / N$, we write it as $g N, g \in \Gamma$. Then $b=g \Delta_{1} \in \Gamma / \Delta_{1}=\widehat{F}$. Let $b^{\prime}=g^{\prime} \Delta_{1}$. Let $\widetilde{w}$ be a representative of $w$ in the normalizer of $T^{\text {aff }}$. Since $w\left(b, b^{\prime}\right)=w$, we can write (uniquely) $g^{\prime}=g n \widetilde{w} \delta$ with $n \in N, \delta \in \Delta_{1}$. We then set $J_{b b^{\prime}}(x)=g n \widetilde{w} N \in \mathcal{T}_{b^{\prime}}$. It is immediate that the $J_{b b^{\prime}}$ are well defined, $\Gamma$-equivariant and satisfy the transitivity conditions. Hence the same is true for the $j_{b b^{\prime}}$.

(b) follows from (a) and (1.4.3).

The purpose of this paper is to make sense of the Hecke algebra of $\Gamma$ by $\Delta_{1}$.

\section{§2. Cherednik algebras}

(2.1) The definitions. We keep the notation of (1.1). As $G$ is assumed simply connected, $L$ is spanned by coroots. Let $Q \subset L^{\vee}$ be the lattice spanned by the roots, so that $L \subset Q^{\vee}$. Note that $\widehat{W}=W \propto L$ while $\widehat{W}_{\text {ad }}=W \propto Q^{\vee}$ is the extended affine Weyl group corresponding to $G_{\text {ad }}$, the adjoint quotient of $G$. Let $m \in \mathbf{Z}_{+}$be the minimal number such that $m \cdot(a, b) \in \mathbf{Z}$ for any $a \in Q^{\vee}, b \in L^{\vee}$. Set

$$
P_{\text {aff }}=L^{\vee} \oplus \frac{1}{m} \mathbf{Z}, \quad \widetilde{T}^{\text {aff }}(\mathbf{C})=\operatorname{Spec} \mathbf{C}\left[P_{\text {aff }}\right]=T(\mathbf{C}) \times \operatorname{Spec} \mathbf{C}\left[\zeta^{ \pm 1 / m}\right] .
$$

Here $\zeta$ is an independent variable which we will think of as the second coordinate on the torus $\widetilde{T}^{\text {aff }}(\mathbf{C})$, writing a typical point of this torus as $\lambda=(\bar{\lambda}, \zeta)$ with $\bar{\lambda} \in T(\mathbf{C})$. The group $\widehat{W}_{\text {ad }}$ acts on $\widetilde{T}^{\text {aff }}(\mathbf{C})$ by

$$
a(\lambda, \zeta)=\left(\zeta^{a} \cdot \lambda, \zeta\right), a \in Q^{\vee}, \quad w(\lambda, \zeta)=(w(\lambda), \zeta), w \in W .
$$

Here the meaning of $\zeta^{a}$ is as follows. To $a$, there corresponds a homomorphism $L^{\vee} \rightarrow \frac{1}{m} \mathbf{Z}$ taking $b \mapsto(b, a)$. To this homomorphism we associate a homomorphism of tori Spec $\mathbf{C}\left[\zeta^{ \pm 1 / m}\right] \rightarrow T$ whose value on $\zeta$ is denoted by $\zeta^{a}$.

Let $\Pi \subset \widehat{W}_{\text {ad }}$ be the subgroup of elements of length 0 . It acts naturally on $P_{\text {aff }}$. 
Let $r \in \mathbf{C}^{*}$ be a fixed nonzero number. The Cherednik algebra $\mathcal{H}=\mathcal{H}_{r}$ associated to the root system of $G$ is, by definition [Ch], generated by the elements

$$
\zeta^{ \pm 1 / m}, \quad \tau_{w}, w \in \widehat{W}, \quad \tau_{\pi}, \pi \in \Pi, \quad Y_{b}, b \in P,
$$

subject to the following relations:

(2.1.3). $\zeta$ is central and the $Y_{b}$ form the group algebra $\mathbf{C}\left[L^{\vee}\right]$, i.e., $Y_{0}=1$ and $Y_{b} Y_{b^{\prime}}=Y_{b b^{\prime}}$; the $\tau_{w}$ and $\tau_{\pi}$ form an affine Hecke algebra of $G_{\text {ad }}$; see, e.g., [GKV]. We abbreviate $\tau_{s_{\alpha}}, \alpha \in R_{+}$, to $\tau_{\alpha}$ and $\tau_{s_{\alpha_{0}}}$ to $\tau_{0}$. We also write $Y_{(b, n)}=Y_{b} \zeta^{n}$ for $(b, n) \in P_{\text {aff }}$.

$$
\begin{gathered}
\tau_{\alpha} Y_{b} \tau_{\alpha}^{-1}=Y_{b} Y_{\alpha}^{-1}, \quad \alpha \in R_{\mathrm{sim}},\left(b, \alpha^{\vee}\right)=1 . \\
\tau_{0} Y_{b} \tau_{0}^{-1}=Y_{b} Y_{\theta}^{-1} \zeta, \quad\left(b, \theta^{\vee}\right)=1 . \\
\tau_{\alpha} Y_{b}=Y_{b} \tau_{\alpha}, \quad \alpha \in R_{\mathrm{sim}},(b, \alpha)=0 . \\
\tau_{\pi} Y_{b} \tau_{\pi}^{-1}=Y_{\pi(b)}, \quad \pi \in \Pi .
\end{gathered}
$$

(2.2) The residue construction. In this paper we will use another construction of the Cherednik algebra which does not use generators and relations. This construction was given in $\mathrm{GKV}$.

For $\alpha=(n, \bar{\alpha}) \in \widehat{R}$, so that $n \in \mathbf{Z}$ and $\bar{\alpha} \in R$, and for $\lambda=(\bar{\lambda}, \zeta) \in \widetilde{T}^{\text {aff }}(\mathbf{C})$ we denote $\lambda^{\alpha}=\zeta^{n} \bar{\lambda}^{\bar{\alpha}}$ and set, for any $z \in \mathbf{C}^{*}$,

$$
\widetilde{T}_{\alpha, z}^{\mathrm{aff}}=\left\{\lambda \in \widetilde{T}^{\mathrm{aff}}(\mathbf{C}): \lambda^{\alpha}=z\right\} .
$$

Let $\mathbf{C}\left(\widetilde{T}^{\text {aff }}\right)$ be the field of rational functions on $\widetilde{T}^{\text {aff }}(\mathbf{C})$. It is acted upon by $\widehat{W}_{\text {ad }}$, via (2.1.2). Let $\mathbf{C}\left(\widetilde{T}^{\text {aff }}\right)\left[\widehat{W}_{\text {ad }}\right]$ be the corresponding twisted group algebra. Its elements are finite formal sums $\sum_{w \in \widehat{W}_{\text {ad }}} f_{w}(t)[w]$, with $f_{w}(t) \in \mathbf{C}\left(\widetilde{T}^{\text {aff }}\right)$ and the multiplication given by the rules:

$$
[w]\left[w^{\prime}\right]=\left[w w^{\prime}\right], \quad[w] f=f^{w}[w], f^{w}(\lambda)=f\left(w^{-1}(\lambda)\right) .
$$

(2.2.3) Theorem. The Cherednik algebra $\mathcal{H}_{r}$ is isomorphic to the subalgebra in $\mathbf{C}\left(\widetilde{T}^{\mathrm{aff}}\right)\left[\widehat{W}_{\mathrm{ad}}\right]$ consisting of $\sum f_{w}(\lambda)[w]$ such that:

(1) The only possible singularities of each $f_{w}$ are first order poles along the $\widetilde{T}_{\alpha, 1}^{\mathrm{aff}}$, $\alpha \in \widehat{R}_{+}$, with

$$
\operatorname{Res}_{\widetilde{T}_{\alpha, 1}^{\text {aff }}}\left(f_{w}\right)+\operatorname{Res}_{\widetilde{T}_{\alpha, 1}^{\text {aff }}}\left(f_{s_{\alpha} w}\right)=0, \quad w \in \widehat{W}, \alpha \in \widehat{R}_{+} .
$$

(2) Each $f_{w}$ vanishes along each $\widetilde{T}_{\alpha, r^{2}}^{\mathrm{aff}}, \alpha \in \widetilde{D}(w)$.

Proof. The statement is almost identical with Theorem 6.3.1 of GKV with one small difference. Namely, the algebra denoted by $\ddot{H}_{r}$ in GKV corresponds to the subalgebra in Cherednik's $\mathcal{H}_{r}$ generated by $\tau_{w}, \tau_{\pi}$ and $Y_{b}$, where $Y_{b}$ runs only over $Q \subset L^{\vee}$. In other words, if we denote by $\breve{G}$ the Langlands dual group of $G$ and by $\check{\Pi}$ the group of length 0 elements in the extended affine Weyl group of its adjoint quotient, then $\mathcal{H}_{r}=\ddot{H}_{r}[\check{\Pi}]$ (the twisted group algebra). Furthermore, the theorem just cited gives the description of $\ddot{H}_{r}$ inside $\mathbf{C}\left(\widetilde{T}^{\text {aff }}\right)[\widehat{W}] \subset \mathbf{C}\left(\widetilde{T}^{\text {aff }}\right)\left[\widehat{W}_{\text {ad }}\right]$ by the same conditions (1) and (2) as in (2.2.3). It remains to notice that

$$
\mathbf{C}\left(\widetilde{T}^{\mathrm{aff}}\right)\left[\widehat{W}_{\mathrm{ad}}\right] \simeq \mathbf{C}\left(\widetilde{T}^{\mathrm{aff}}\right)[\widehat{W}][\check{\Pi}]
$$


and that the validity of conditions (1)-(2) for an element $\phi$ in the LHS is equivalent to their validity for any coefficient $\phi_{\omega} \in \mathbf{C}\left(\widetilde{T}^{\text {aff }}\right)[\widehat{W}]$ in the decomposition $\phi=$ $\sum_{\omega \in \check{\Pi}} \phi_{\omega}[\omega]$ in the RHS.

(2.3) The modified Cherednik algebra. Consider the embedding of lattices

$$
L_{\text {aff }}=L \oplus \mathbf{Z} \stackrel{\Psi \oplus \mathrm{Id}}{\hookrightarrow} P_{\text {aff }}=L^{\vee} \oplus \frac{1}{m} \mathbf{Z}
$$

where $\Psi$ is the form (1.1.1), and the corresponding homomorphism (finite covering) of tori

$$
\widetilde{T}^{\mathrm{aff}}(\mathbf{C})=\operatorname{Spec} \mathbf{C}\left[P_{\mathrm{aff}}\right] \rightarrow \operatorname{Spec} \mathbf{C}\left[L_{\mathrm{aff}}\right]=\check{T}^{\mathrm{aff}} .
$$

The above maps are $W$-equivariant. Therefore the action $(2.1 .2)$ of $\widehat{W}_{\text {ad }}$ on $\widetilde{T}^{\text {aff }}(\mathbf{C})$, being composed of the standard $W$-action and of the action of $Q^{\vee}$ by translations, descends to an action on $\check{T}^{\text {aff }}$. Thus the subfield $\mathbf{C}\left(\check{T}^{\text {aff }}\right) \subset \mathbf{C}\left(\widetilde{T}^{\text {aff }}\right)$ is preserved

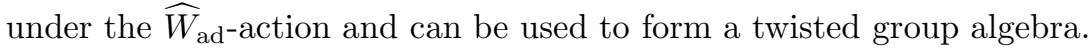

(2.3.3) Definition. The modified Cherednik algebra $\ddot{H}_{r}(G)$ is the intersection

$$
\mathbf{C}\left(\check{T}^{\mathrm{aff}}\right)[\widehat{W}] \cap \mathcal{H}_{r} \subset \mathbf{C}\left(\widetilde{T}^{\text {aff }}\right)\left[\widehat{W}_{\text {ad }}\right] .
$$

\section{§3. Hecke operators. Main theorem}

(3.1) Generalities. Let $\Gamma$ be a group and $\Delta \subset \Gamma$ a subgroup. We will denote by $\Gamma / / \Delta$ the set of double cosets of $\Gamma$ by $\Delta$. For any such coset $C=\Delta \gamma \Delta$ we have the Hecke correspondence $\Sigma_{C}$ which is the subset

$$
\Sigma_{C}=\left\{\left(\gamma_{1} \Delta, \gamma_{2} \Delta\right) \mid \gamma_{2} \gamma_{1}^{-1} \in C\right\} \quad \subset \quad(\Gamma / \Delta) \times(\Gamma / \Delta) .
$$

The $\Sigma_{C}$ are nothing else than all the $\Gamma$-orbits on $(\Gamma / \Delta) \times(\Gamma / \Delta)$. For $x \in \Gamma / \Delta$ we denote by $\Sigma_{C}(x)$ the intersection $\Sigma_{C} \cap((\Gamma / \Delta) \times\{x\})$. Denote also

$$
\Gamma / \Delta \stackrel{p_{1}}{\longleftarrow} \Sigma_{C} \stackrel{p_{2}}{\rightarrow} \Gamma / \Delta
$$

to be the natural projections. Thus $\Sigma_{C}(x)=p_{2}^{-1}(x)$.

If $\Gamma$ is a locally compact Hausdorff topological group and $\Delta$ is a compact subgroup, then a Haar measure on $\Gamma$ induces natural measures $\mu_{C, x}$ on each $\Sigma_{C}(x)$ invariant under $\operatorname{Stab}(x) \subset \Gamma$. Denoting by $\mathcal{F}(\Gamma / \Delta)$ the space of continuous functions $\Gamma / \Delta \rightarrow \mathbf{C}$, we have the $\Gamma$-invariant operator

$$
\tau_{C}: \mathcal{F}(\Gamma / \Delta) \rightarrow \mathcal{F}(\Gamma / \Delta), \quad\left(\tau_{C} f\right)(x)=\int_{y \in \Sigma_{C}(x)} f(y) d \mu_{C, x},
$$

known as the Hecke operator. Under our assumptions, each $\Sigma_{C}(x)$ is compact, so the integral makes sense. The Hecke algebra $H(\Gamma, \Delta)$ is the convolution algebra of continuous compactly supported, $\Delta$-biinvariant functions on $\Gamma$. Its elements can be thought of as (continuous) linear combinations of the operators $\tau_{C}$.

We now want to apply the above formalism to the case when $\Gamma$ is the central extension of $G(K)$ introduced in (1.3), and $\Delta=\Delta_{1}$ is the "intermediate Iwahori subgroup". In this case, the natural topology on $K$ is not locally compact and, moreover, fails to make it into a topological ring ([FP], Kat $)$, so there is no obvious good topology on $G(K)$ and $\Gamma$ and no Haar measure. Nevertheless, $\Xi=\Gamma / \Delta_{1}$ is an

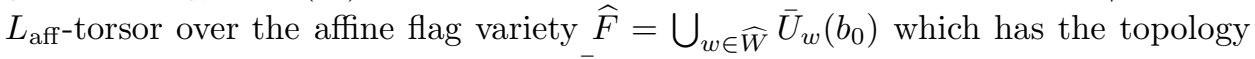
of inductive limit of compact spaces $\bar{U}_{w}\left(b_{0}\right)$. From now on we will freely use the formalism and notation set up in the Appendix, as well as those of $\S 1$. 
(3.2) Function spaces associated to a p-adic loop group. The space $\widehat{F}$ is an object of $\mathcal{K}$ and will also be regarded as an ind-pro-object of $\mathcal{S}_{0}$ (with an action of $G(K)$ by isomorphisms).

(3.2.1) Proposition. The set-theoretic $L_{\text {aff-torsor }} \Xi \rightarrow \widehat{F}$ has a natural structure of a $\Gamma$-equivariant object of the category $L_{\mathrm{aff}}-\operatorname{Tors}(\widehat{F})$.

Proof. It is enough to show that for any homomorphism $\chi: L_{\text {aff }} \rightarrow \mathbf{Z}$ the induced set-theoretical torsor $\chi_{*} \Xi \rightarrow \widehat{F}$ has a natural structure of a $\Gamma$-equivariant object of Z-Tors $(\widehat{F})$.

Let $\Pi$ be the category of projective algebraic varieties over $k$. Taking the space of $k$-points defines a functor $\gamma: \Pi \rightarrow \mathcal{P}$. The induced functor on ind-objects will also be denoted $\gamma: \operatorname{Ind}_{s}^{\aleph_{0}}(\Pi) \rightarrow \mathcal{K}$. For a variety $M \in \Pi$ let $\operatorname{Bun}_{1}^{\text {alg }}(M)$ be the category of algebraic line bundles on $M$, and for an object $N=\left(N_{i}\right)$ of $\operatorname{Ind}_{s}^{\aleph_{0}}(\Pi)$ we

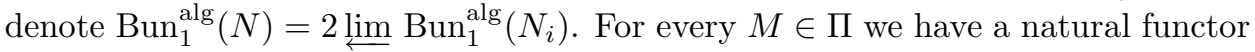

$$
\operatorname{Bun}_{1}^{\text {alg }}(M) \rightarrow k-\operatorname{Bun}_{1}(\gamma M)
$$

(the topological bundle on $k$-points induced by an algebraic bundle). For every $Y \in \mathcal{P}$ we have a natural functor

$$
\operatorname{ord}_{*}: k-\operatorname{Bun}_{1}(Y) \rightarrow \mathbf{Z} \text {-Tors }(Y), \quad E \mapsto(E-\{0\}) / \mathcal{O}_{k}^{*} .
$$

Composing these functors and passing to ind-objects, we get, for $N \in \operatorname{Ind}_{s}^{\aleph_{0}}(\Pi)$, a natural functor

$$
\delta: \operatorname{Bun}_{1}^{\text {alg }}(N) \rightarrow \mathbf{Z} \text {-Tors }(\gamma N) .
$$

Now, $\widehat{F}$ comes from an object $\underline{\widehat{F}}$ of $\operatorname{Ind}_{s}^{\aleph_{0}}(\Pi)$, in the sense that $\widehat{F}=\gamma \underline{\widehat{F}}$. Further, it is well known that $\chi$ gives rise to an object $\mathcal{O}(\chi) \in \operatorname{Bun}_{1}^{\text {alg }}(\underline{\widehat{F}})$, equivariant under $\Gamma$. It remains to notice that $\chi_{*} \Xi=\delta(\mathcal{O}(\chi))$. The proposition is proved.

Thus we can speak about the function spaces $\mathcal{F}_{0}(\Xi), \mathcal{F}(\Xi)$, etc. Let $L_{\text {aff }}^{+} \subset L_{\text {aff }}$ be the convex cone spanned by the positive affine roots. For any element $w \in \widehat{W}$ let $L_{\text {aff }}^{w}=w\left(L_{\text {aff }}^{+}\right)$. We define $\mathcal{F}_{w}(\Xi)=\mathcal{F}_{L_{\text {rat }}^{w}}(\Xi)$ and similarly define $\mathcal{F}_{w}^{\text {rat }}(\Xi)$.

(3.3) Hecke operators and the main theorems. Let $(w, l) \in \widetilde{W}=\Gamma / / \Delta_{1}$ (see (1.4.6)), and let $\Sigma_{w, l} \subset \Xi \times \Xi$ be the Hecke correspondence ( $\Gamma$-orbit) associated to $(w, l)$. For $\xi \in \Xi$ let $\Sigma_{w, l}(\xi) \subset \Xi$ consist of $\xi^{\prime}$ such that $\left(\xi, \xi^{\prime}\right) \in \Sigma_{w, l}$. By (1.4.6), this is an affine space over $k$ of dimension $l(w)$. The stabilizer $\operatorname{Stab}(\xi) \subset \Gamma$ acts transitively on $\Sigma_{w, l}(\xi)$.

(3.3.1) Proposition. For every $\xi$ the space of complex valued Borel measures on $\Sigma_{w, l}(\xi)$ invariant under $\operatorname{Stab}(\xi)$ is nonzero (and hence is 1-dimensional).

Proof. It is enough to take $\xi=\xi_{0}$, the distinguished point of $\Xi=\Gamma / \Delta_{1}$, so that $\operatorname{Stab}(\Xi)=\Delta_{1}$. In this case the action of $\Delta_{1}$ on $\Sigma_{w, l}(\Xi)$ factors through $\Delta_{1} / \Gamma_{0}(N)$, where $N \gg 0$ is a sufficiently large integer and

$$
\Gamma_{0}(N)=\left\{g \in G\left(\mathcal{O}_{K}\right): g \equiv 1 \bmod \mathbf{m}_{K}^{N}\right\}
$$

is the principal congruence subgroup of level $N$. The quotient $\Delta_{1} / \Gamma_{0}(N)$ is an extension

$$
1 \rightarrow E \rightarrow \Delta_{1} / \Gamma_{0}(N) \rightarrow T\left(\mathcal{O}_{k}\right) \rightarrow 1,
$$


where $E$ is the group of $k$-points of a unipotent algebraic group over $k$. Further, $\Delta_{1} / \Gamma_{0}(N)$ acts on the affine space $\Sigma_{w, l}(\xi)$ by polynomial transformations, and the action of $E$ comes from an algebraic action of an algebraic group. It follows from this and from the compactness of $T\left(\mathcal{O}_{k}\right)$ that for every $g \in \Delta_{1} / \Gamma_{0}(N)$ and any $g$-fixed point $\eta \in \Sigma_{w, l}(\xi)$ the jacobian determinant $\operatorname{det}\left(d_{\eta} g\right) \in k^{*}$ has $k$-absolute value 1 . This implies the proposition.

We conlude (by using translation) that a choice of a $\operatorname{Stab}(\xi)$-invariant measure $\mu$ on $\Sigma_{w, l}(\xi)$ for some one $\xi$ defines unambiguously a measure $\mu_{\xi^{\prime}}$ on $\Sigma_{w, l}\left(\xi^{\prime}\right)$ for any $\xi^{\prime}$. Notice also that for any $l, l^{\prime} \in L_{\text {aff }}$ the spaces $\Sigma_{w, l}(\xi)$ and $\Sigma_{w, l^{\prime}}(\xi)$ are canonically identified (1.4.6).

So for every $w \in \widehat{W}$ we choose in some way an invariant measure $\mu_{w, 0, \xi_{0}}$ on $\Sigma_{w, 0}\left(\xi_{0}\right)$ and then define the measure $\mu_{w, l, \xi}$ on $\Sigma_{w, l}(\xi)$ by using the above identifications. Thus, for a continuous function $f: \Xi \rightarrow \mathbf{C}$ we can formally write the integral

$$
\left(\tau_{w, l} f\right)(\xi)=\int_{\eta \in \Sigma_{w, l}(\xi)} f(\eta) d \mu_{w, l, \xi}
$$

defining the Hecke operator. Since the domain of integration is noncompact, the integral may not converge. If $w=e$, the integration is over a point and $\tau_{e, l}$ is the (well-defined) operator of shift by $l$. We now state three theorems describing the regularization of the $\tau_{w, l}$, of their compositions and the structure of the algebra formed by the regularized operators. The proofs will be given in the next section.

(3.3.3) Theorem. If $f \in\left|\mathcal{F}_{0}(\Xi)\right|$, then $\operatorname{Supp}(f) \cap \Sigma_{w, l}(\xi)$ is compact for any $w, l, \xi$, so the integral (3.3.2) converges and gives rise to a well-defined morphism (Hecke operator)

$$
\tau_{w, l}: \mathcal{F}_{0}(\Xi) \rightarrow \mathcal{F}(\Xi) \quad \in \quad \operatorname{Mor}\left(\operatorname{Pro}\left(\operatorname{Mod}_{\mathbf{C}[L]}\right)\right) .
$$

In particular, $\tau_{e, l}$ is the shift by $l$ (preserving $\mathcal{F}_{0}(\Xi)$ ) and $\tau_{w, l+l^{\prime}}=\tau_{w, l^{\prime}} \tau_{e, l}$.

For $l \in L_{\text {aff }}$ the corresponding element of $\mathbf{C}\left[L_{\text {aff }}\right]$ will be denoted $t^{l}$, so that a generic element will be written as a Laurent polynomial $f(t)=\sum_{l} a_{l} t^{l}$. The operator $\tau_{w, 0}$ will be abbreviated to $\tau_{w}$. Let $H_{\text {pol }}\left(\Gamma, \Delta_{1}\right)$ be the space of formal finite $\mathbf{C}$-linear combinations

$$
\sum_{w, l} a_{w, l} \tau_{w, l}=\sum_{w \in \widehat{W}} f_{w}(t) \tau_{w}, \quad a_{w, l} \in \mathbf{C}, f_{w} \in \mathbf{C}\left[L_{\mathrm{aff}}\right],
$$

of the Hecke operators corresponding to elements of $\Gamma / / \Delta_{1}$. This space is not yet an algebra since the $\tau_{w, l}$ act from one space to another. It is, however, a $\mathbf{C}\left[L_{\text {aff }}\right]-$ module and we get a $\mathbf{C}\left[L_{\text {aff }}\right]$-linear map

$$
H_{\text {pol }}\left(\Gamma, \Delta_{1}\right) \rightarrow \operatorname{Hom}_{\operatorname{Pro}\left(\operatorname{Ind}\left(\operatorname{Mod}_{\mathbf{C}\left[L_{\text {aff }}\right]}\right)\right)}\left(\mathcal{F}_{0}(\Xi), \mathcal{F}(\Xi)\right) .
$$

Recall that the torus Spec $\mathbf{C}\left[L_{\text {aff }}\right]$ is denoted $\check{T}_{\text {aff }}$, so $\mathbf{C}\left(\check{T}_{\text {aff }}\right)$ is the field of fractions of $\mathbf{C}\left[L_{\text {aff }}\right]$.

(3.3.5) Theorem. The operator $\tau_{w, l}$ from (3.3.3) takes values in $\mathcal{F}_{w}^{\text {rat }}(\Xi) \subset \mathcal{F}(\Xi)$ and gives rise to an operator

$$
\tau_{w, l}^{\mathrm{rat}} \in \operatorname{End}_{\operatorname{Pro}\left(\operatorname{Mod}_{\mathbf{C}\left(\check{T}_{\text {aff }}\right)}\right)}\left(\mathcal{F}^{\mathrm{rat}}(\Xi)\right)
$$


fitting into the commutative diagram in $\operatorname{Pro}\left(\operatorname{Ind}\left(\operatorname{Mod}_{\mathbf{C}\left[L_{\text {aff }}\right]}\right)\right)$ :

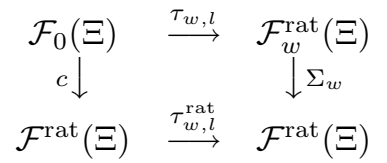

where $c$ is the canonical embedding and $\Sigma_{w}$ is the summation map from (A.5.2).

The operator $\tau_{w}^{\text {rat }}$ can be thought of as a regularized Hecke operator. It now acts from a vector space to itself. This is achieved by a regularization procedure consisting in summation of a series to a rational function and re-expansion in a different domain. So it now makes sense to ask about the composition of the $\tau_{w, l}^{\text {rat }}$. As before, set $\tau_{w}^{\text {rat }}=\tau_{w, 0}^{\text {rat }}$. Let

$$
H_{\text {rat }}\left(\Gamma, \Delta_{1}\right)=\mathbf{C}\left(\check{T}_{\text {aff }}\right) \otimes_{\mathbf{C}\left[L_{\text {aff }}\right]} H_{\text {pol }}\left(\Gamma, \Delta_{1}\right)=\left\{\sum_{w \in \widehat{W}} f_{w}(t) \tau_{w}^{\mathrm{rat}} \mid f_{w} \in \mathbf{C}\left(\check{T}_{\mathrm{aff}}\right)\right\}
$$

be the space of formal $\mathbf{C}\left(\check{T}_{\text {aff }}\right)$-linear combinations of the $\tau_{w}^{\text {rat }}$. The map (3.3.4) induces a $\mathbf{C}\left(\check{T}_{\text {aff }}\right)$-linear map

$$
\tau: H_{\text {rat }}\left(\Gamma, \Delta_{1}\right) \rightarrow \operatorname{End}_{\operatorname{Pro}\left(\operatorname{Mod}_{\mathbf{C}\left[L_{\text {aff }}\right]}\right)}\left(\mathcal{F}^{\text {rat }}(\Xi)\right) .
$$

Expanding the rational functions $f_{w}$ into power series in some domain, we can view elements of $H_{\text {rat }}\left(\Gamma, \Delta_{1}\right)$ as certain infinite formal linear combinations of the operators corresponding to double cosets. It turns out that considering such combinations is necessary for the algebra generated by the $\tau_{w}^{\text {rat }}$ to close.

(3.3.8) Theorem. (a) The space $H_{\text {rat }}\left(\Gamma, \Delta_{1}\right)$ has a natural structure of an associative algebra so that $\tau$ is a homomorphism of algebras.

(b) Let $H\left(\Gamma, \Delta_{1}\right) \subset H_{\text {rat }}\left(\Gamma, \Delta_{1}\right)$ be the subalgebra formed by $S$ such that $\tau(A)$ preserves $\mathcal{F}_{0}(\Xi) \subset \mathcal{F}^{\mathrm{rat}}(\Xi)$. Then $H\left(\Gamma, \Delta_{1}\right)$ is isomorphic to the modified Cherednik algebra $\ddot{H}_{q}(G)$ from (2.3.3).

(3.3.9) Remarks. (a) The above approach can be applied to the case of the locally compact group $G(k)$ and the noncompact subgroup $N(k) T\left(\mathcal{O}_{k}\right)$. In this case the properties of p-adic intertwiners together with Theorem 5.4 of [GKV] can be used to give a simple proof of the main result of [CK].

(b) The shape of the particular infinite combinations of double cosets appearing in the Hecke algebra $H\left(\Gamma, \Delta_{1}\right)$ and in the simpler situation of Remark (a) is remindful of the procedure of perverse (or intersection homology) extension of sheaves. The right context here seems to be the (not yet developed) theory of "semi-infinite" perverse extension as sketched by Lusztig [Lu2].

(3.3.10) Corollary. For any subgroup $U \subset \Gamma$ the (pro-) space of invariants $\mathcal{F}_{0}(\Xi)^{U}$ is naturally acted upon by $\ddot{H}_{q}(G)$.

(3.3.11) Example. An interesting example of a subgroup $U \subset \Gamma$ is obtained as follows. Let $C$ be a smooth irreducible projective curve over $k$, and let $x \in C$ be a $k$ point. Let $K=k(C)_{x}$ be the completion of $k(C)$ at $x$. Then $K \simeq k((t))$, so $(3.3 .9)$ is applicable to $U:=G(k[C-\{x\}]) \subset \Gamma$. We get an action of $\ddot{H}_{q}(G)$ on $\left|\mathcal{F}_{0}(\Xi)\right|^{U}$. Elements of the latter space can be viewed as certain functions on isomorphism classes of principal $G$-bundles on $C$ equipped with a "horocyclic structure" (i.e., 
with a reduction of the structure group from $G(k)$ to $\left.N(k) T\left(\mathcal{O}_{k}\right)\right)$ at $x$ and with a choice of a nonzero vector in the determinantal space det $H^{\bullet}(C, \operatorname{ad}(P))$. We get in this way a setup for generalizing the theory of automorphic forms over function fields [Dr] from the case of a finite to the case of a p-adic field of constants. Hecke operators are now (linear combinations of) singular integral operators, while in the classical case they are given by finite summation.

\section{§4. Principal series Representations AND THE PROOF OF THE MAIN THEOREMS}

(4.1) The unramified principal series. We specialize the discussion of (A.7) to

$$
A=L_{\text {aff }}, \quad T_{A}=\check{T}_{\text {aff }}, \quad Z=\widehat{F}, \quad B=\Xi ;
$$

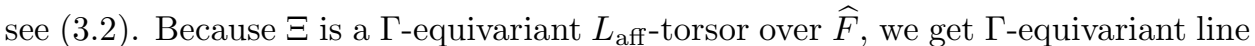
bundles $\mathcal{L}(\lambda), \lambda \in \check{T}_{\text {aff }}$, on $\widehat{F}$. The "space" of sections $V_{\lambda}=\Gamma(\widehat{F}, \mathcal{L}(\lambda))$ is acted upon by $\Gamma$. It will be called the unramified principal series representation of $\Gamma$. According to our general principles, we consider it as an object of Pro(Vect).

The pro- $\mathbf{C}\left(\check{T}_{\text {aff }}\right)$-vector space $\Gamma_{\text {rat }}\left(\check{T}_{\text {aff }}, V\right)$ will be called the generic principal series representation. The Mellin transform from (A.7) gives identifications

$$
\mathcal{F}_{0}(\Xi) \rightarrow \Gamma_{\text {reg }}\left(\check{T}_{\text {aff }}, V\right), \quad \mathcal{F}^{\text {rat }}(\Xi) \rightarrow \Gamma_{\text {rat }}\left(\check{T}_{\text {aff }}, V\right) .
$$

For $b \in \widehat{F}$ let $I_{b} \subset \Gamma$ be the stabilizer of $b$ and $N_{b}=\left[I_{b}, I_{b}\right]$. For $w \in \widehat{W}$ denote by $\mu_{w}(b)$ the vector space of $N_{b}$-invariant $\mathbf{C}$-valued Borel measures on the Schubert cell $U_{w}(b)$. By the same reason as in (3.3.1), we have $\operatorname{dim}_{\mathbf{C}}\left(\mu_{w}(b)\right)=1$.

(4.1.2) Proposition. (a) When $w \in \widehat{W}$ is fixed, the $\mu_{w}(b)$ form the fibers of a $\Gamma$-equivariant line bundle $\mu_{w}$ on $\widehat{F}$ in the sense of (A.4.2).

(b) The bundle $\mu_{w}$ is $\Gamma$-equivariantly isomorphic to $\mathcal{L}\left(q^{\widehat{\delta}_{w}}\right)$, where $\widehat{\delta}_{w}$ was defined in (1.1.6).

Proof. (a) Let $U_{w} \in \widehat{F} \times \widehat{F}$ be the Schubert correspondence, and let $p_{w}: U_{w} \rightarrow \widehat{F}$ be the projection onto the second factor. The fact that the $\mu_{w}(b)$ tie together to form a line bundle on $\widehat{F}$ in the sense of ind-objects in $\operatorname{Pro}\left(\mathcal{S}_{0}\right)$ follows from the fact that $p_{w}$ gives rise to a locally trivial algebraic affine bundle over $\widehat{F}$ in the sense of ind-objects in the category of projective $k$-varieties. This latter fact is well known.

(b) It is enough to consider the distinguished point $b_{0} \in \widehat{F}$ with stabilizer $I_{K}$. Then, $U_{w}\left(b_{0}\right)$ has a unique $I_{K}$-fixed point $b_{w}$ and the eigenvectors of the $I_{K}$-action on $T_{b_{w}} U_{w}\left(b_{0}\right)$ are in bijection with positive affine roots from $D(w)$, whence the statement.

We now proceed to define the analogs, in our affine situation, of the principal series intertwiners for p-adic groups ([GGP, $[\overline{\mathrm{Cas}}])$. Consider the action (1.1.3) of $\widehat{W}$ on $L_{\text {aff }}$; the induced action on $\check{T}_{\text {aff }}=\operatorname{Spec} \mathbf{C}\left[L_{\text {aff }}\right]$ will be denoted by $\lambda \mapsto w(\lambda)$. Introduce the twisted $\widehat{W}$-action on $\check{T}_{\text {aff }}$ by

$$
w * \lambda=q^{\widehat{\rho}} \cdot w\left(q^{-\widehat{\rho}} \cdot \lambda\right)=q^{\widehat{\delta}_{w}} \cdot w(\lambda)=w\left(q^{-\widehat{\delta}_{w}} \cdot \lambda\right) .
$$

Define the linear operator

$$
M_{w}(\lambda): V_{\lambda}=\Gamma\left(\widehat{F}, \mathcal{L}\left(\lambda q^{-\widehat{\delta}_{w}}\right) \otimes \mu_{w}\right) \rightarrow \Gamma(\widehat{F}, \mathcal{L}(w * \lambda))=V_{w * \lambda}
$$


by

$$
M_{w}(\lambda)(f \otimes m)(\xi)=\int_{b^{\prime} \in U_{w}(b)} f\left(j_{b b^{\prime}}(\xi)\right) d m\left(b^{\prime}\right), \quad \xi \in \Xi_{b}
$$

(provided the integral converges). Note that up to the choice of the class of functions considered, (4.1.5) is identical with the formula (3.3.2) defining the Hecke operator $\tau_{w, 0}$

(4.1.6) Proposition. (a) If $\left|\lambda^{\alpha^{\vee}}\right|>q^{-1}$ for any $\alpha \in D(w)$, then the integral (4.1.5) converges and gives rise to a $\Gamma$-equivariant morphism $M_{w}(\lambda): V_{\lambda} \rightarrow V_{w * \lambda}$ in Pro(Vect).

(b) The operators $M_{w}(\lambda)$ extend to a rational, in the sense of (A.6.2), isomorphism $M_{w}: V \rightarrow w^{*} V$ of pro-vector bundles on $\check{T}_{\text {aff }}$.

(c) The operators

$$
A_{w}=\frac{1}{c_{w}(\lambda)} M_{w}, \quad c_{w}(\lambda)=\prod_{\alpha \in D(w)} \frac{1-\lambda^{\alpha^{\vee}}}{1-q \lambda^{\alpha^{\vee}}},
$$

satisfy the conditions $A_{w} A_{w^{\prime}}=A_{w w^{\prime}}$, in particular, they form a representation of $\widehat{W}$ in $V_{\text {rat }}=\Gamma_{\text {rat }}\left(\check{T}_{\text {aff }}, V\right)$.

(d) The only singularities, in the sense of (A.6.3), of $A_{w}$ are first order poles at the hypersurfaces $\check{T}_{\alpha, 1}^{\mathrm{aff}}, \alpha \in D(w)$.

The reader is referred to Section A.6 of the Appendix for background on rational morphisms of pro-vector bundles and their singularities.

Proof. The argument is similar to the proof of the analogous statement (see GGP, $\mathrm{Cas}$ ) for the locally compact group $G(k)$; the new phenomenon is that we have to deal with pro-vector bundles rather than usual vector bundles.

Step 1: the case of a simple reflection. We first establish the proposition in the case when $w$ is a simple affine reflection: $w=s_{\alpha}, \alpha \in \widehat{R}_{\text {sim. }}$. Let $P_{\alpha}$ be the parahoric subgroup in $\Gamma$ corresponding to the set of roots $\widehat{R}_{+} \cup\{-\alpha\}$, so that we have the projection

$$
p_{\alpha}: \widehat{F} \rightarrow \widehat{F}_{\alpha}:=\Gamma / P_{\alpha}
$$

with fibers isomorphic to $P^{1}(k)$.

If $Z \subset \widehat{F}$ is a closed subscheme, then we have a vector space $V_{\lambda}(Z):=\Gamma(Z, \mathcal{L}(\lambda))$ and a vector bundle (possibly of infinite rank) $V(Z)$ on $\check{T}^{\text {aff }}$ with fibers $V_{\lambda}(Z)$; see (A.7). The ind-scheme structure on $\widehat{F}$ is given in terms of the affine Schubert varieties:

$$
\widehat{F}=“ \lim ^{\longrightarrow} \widehat{W}_{y \in \bar{U}_{y}}\left(b_{0}\right),
$$

where $b_{0} \in \widehat{F}$ is some fixed reference point. Accordingly, the pro-vector space structure on $V_{\lambda}$ and the pro-vector bundle structure on $V$ are given explicitly by

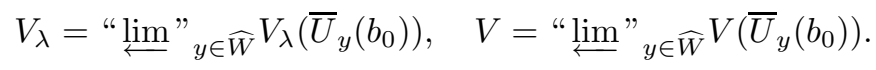

Our first step is to replace (4.1.8) by a different realization of $\widehat{F}$ as an inductive limit of projective varieties which is more adapted to our situation. Namely, let $\widehat{W}_{\alpha}=W /\left\{1, s_{\alpha}\right\}$. This set parametrizes $G(K)$-orbits in $\widehat{F} \times \widehat{F}_{\alpha}$. Let $U_{z}, z \in \widehat{W}_{\alpha}$, be the orbit corresponsing to $z$ and for $b \in \widehat{F}$ let $U_{z}(b)=U_{z} \cap\left(\{b\} \times \widehat{F}_{\alpha}\right)$. This 
is an affine Schubert cell in $\widehat{F}_{\alpha}$ and we denote by $\bar{U}_{z}(b)$ its closure. This is a projective variety over $k$ and the natural ind-scheme structure on $\widehat{F}_{\alpha}$ is $\widehat{F}_{\alpha}=$ “lim" $z \in \widehat{W}_{\alpha} \bar{U}_{z}\left(b_{0}\right)$. This means that, set-theoretically, $\widehat{F}=\underline{\lim }_{z \in \widehat{W}_{\alpha}} p_{\alpha}^{-1}\left(\bar{U}_{z}\left(b_{0}\right)\right)$; note that each $p_{\alpha}^{-1}\left(\bar{U}_{z}\left(b_{0}\right)\right)$ is again a projective variety over $k$, a $P^{1}$-fibration over $\bar{U}_{z}\left(b_{0}\right)$.

(4.1.10) Lemma. The identity morphism of $\widehat{F}$ lifts to an isomorphism of indschemes

$$
\text { “ } \lim _{y \in \widehat{W}} \bar{U}_{y}\left(b_{0}\right) \rightarrow \text { “ } \lim " \widehat{W}_{\alpha} p_{\alpha}^{-1}\left(\bar{U}_{z}\left(b_{0}\right)\right) .
$$

In plain words, this means that the structures of an ind-scheme on $\widehat{F}$ given by the two inductive systems above are equivalent.

Proof. It is enough to show that the two inductive systems of subschemes in $\widehat{F}$ are cofinal with respect to each other, i.e., for any $y \in \widehat{W}$ there is $z \in \widehat{W}_{\alpha}$ such that $\bar{U}_{y}\left(b_{0}\right) \subset p_{\alpha}^{-1}\left(\bar{U}_{z}\left(b_{0}\right)\right)$ and, conversely, for any $z \in \widehat{W}_{\alpha}$ there is $y \in \widehat{W}$ such that $p_{\alpha}^{-1}\left(\bar{U}_{z}\left(b_{0}\right)\right) \subset \bar{U}_{y}\left(b_{0}\right)$. But this is immediate: in the first case $z$ is the class of $y$ in $\widehat{W}_{\alpha}=W /\left\{1, s_{\alpha}\right\}$, and in the second case $y$ is the supremum (in the Bruhat order) of the two preimages of $z$ in $\widehat{W}$.

(4.1.11) Corollary. The pro-vector space structure on $V_{\lambda}$ and the pro-vector bundle structure on $V$ can be defined as follows:

$$
V_{\lambda}=“ \varliminf{ }_{z \in \widehat{W}_{\alpha}} V_{\lambda}\left(p_{\alpha}^{-1} \bar{U}_{z}\left(b_{0}\right)\right), \quad V=“ \varliminf{ }_{z \in \widehat{W}_{\alpha}} V\left(p_{\alpha}^{-1} \bar{U}_{z}\left(b_{0}\right)\right) .
$$

Now note that the Schubert correspondence $U_{s_{\alpha}} \subset \widehat{F} \times \widehat{F}$ consists of $\left(b, b^{\prime}\right)$ such that $p_{\alpha}(b) \neq p_{\alpha}\left(b^{\prime}\right)$. This means that the action of $M_{s_{\alpha}}$ is fiberwise with respect to $p_{\alpha}$. The action on (the space of sections of $\mathcal{L}(\lambda)$ over) each fiber (isomorphic to $P^{1}(k)$ ) is the same as the action of the principal series intertwiner for the group $S L_{2}(k)$ for which $P^{1}(k)$ is the flag variety. Applying this observation to the fibers of the projection $p_{\alpha}^{-1} \bar{U}_{z}\left(b_{0}\right) \rightarrow \bar{U}_{z}\left(b_{0}\right)$, we deduce (from the well-known properties of $S L_{2}(k)$ ) that we have a linear operator

$$
M_{s_{\alpha}}(\lambda, z): V_{\lambda}\left(p_{\alpha}^{-1} \bar{U}_{z}\left(b_{0}\right)\right) \rightarrow V_{s_{\alpha} * \lambda}\left(p_{\alpha}^{-1} \bar{U}_{z}\left(b_{0}\right)\right)
$$

defined by an integral converging for $\left|\lambda^{\alpha^{\vee}}\right|>q^{-1}$, and a rational isomorphism of vector bundles on $\check{T}^{\text {aff }}$

$$
M_{s_{\alpha}}(z): V\left(p_{\alpha}^{-1} \bar{U}_{z}\left(b_{0}\right)\right) \rightarrow s_{\alpha}^{*} V\left(p_{\alpha}^{-1} \bar{U}_{z}\left(b_{0}\right)\right)
$$

so that $A_{s_{\alpha}}(z)=M_{s_{\alpha}}(z) / c_{s_{\alpha}}(\lambda)$ satisfies $A_{s_{\alpha}}(z)^{2}=1$ and has first order pole along $\check{T}_{\alpha, 1}^{\text {aff }}$.

Now, letting $z \in \widehat{W}_{\alpha}$ vary, we see that the operators $M_{s_{\alpha}}(\lambda, z)$ (resp. rational isomorphisms $\left.M_{s_{\alpha}}(z)\right)$ form a morphism of the inverse system of vector spaces (resp. of vector bundles) from Corollary 4.1.11. So they give rise to a morphism of pro-vector spaces (resp. a rational isomorphism of pro-vector bundles) with the required properties. This establishes the simple reflection case.

Step 2: the general case. Let $w \in \widehat{W}$ be arbitrary, and let $w=s_{\alpha_{1}} \ldots s_{\alpha_{n}}, n=$ $l(w)$, be a reduced decomposition of $w$. Then the properties of the BN-pair associated to the affine root system imply that

$$
U_{w}=U_{s_{\alpha_{1}}} \times_{\widehat{F}} U_{s_{\alpha_{2}}} \times{ }_{\widehat{F}} \ldots \times_{\widehat{F}} U_{s_{\alpha_{n}}}
$$


and thus $M_{w}=M_{s_{\alpha_{1}}} \circ \ldots \circ M_{s_{\alpha_{n}}}$. Because each $M_{s_{\alpha_{i}}}$ is a rational isomorphism (with respect to the pro-vector bundle structure (4.1.9) which is equivalent to that of Corollary 4.1.11), we deduce that $M_{w}$ is a rational isomorphism and $A_{w}$ satisfies the claimed condition on singularities. Finally, if $w=w_{1} w_{2}$ with $l(w)=l\left(w_{1}\right)+l\left(w_{2}\right)$, then $U_{2}=U_{w_{1}} \times_{\widehat{F}} U_{w_{2}}$, so $M_{w}=M_{w_{1}} \circ M_{w_{2}}$ and $A_{w}=A_{w_{1}} \circ A_{w_{2}}$. This, together with $A_{s_{\alpha}}^{2}=1, \alpha \in \widehat{R}_{\text {sim }}$, implies that $A_{w_{1} w_{2}}=A_{w_{1}} \circ A_{w_{2}}$ for any $w_{1}, w_{2}$. Proposition 4.1.6 is proved.

(4.2) Proof of Theorem 3.3.3. We denote by $p: \Xi \rightarrow \widehat{F}$ the projection. Let us equip $\Xi$ with the topology of the inductive limit of locally compact spaces ( $L_{\text {aff- }}{ }^{-}$ torsors) $\Xi^{(y)}=p^{-1}\left(\bar{U}_{y}\left(b_{0}\right)\right), y \in \widehat{W}$. Then any $\Sigma_{w, l}(\xi)$ is contained in some $\Xi^{(y)}$. Every $f \in\left|\mathcal{F}_{0}(\Xi)\right|$ gives, upon restriction to any $\Xi^{(y)}$, a function which is compactly supported in the topological sense. Thus the convergence of $\left(\tau_{w, l} f\right)(\xi)$ would follow from the next lemma.

(4.2.1) Lemma. Each $\Sigma_{w, l}(\xi)$ is closed in $\Xi$ (and hence in any $\Xi^{(y)}$ containing $\left.\Sigma_{w, l}(\Xi)\right)$.

Proof. It is enough to consider the case $l=0$. Denote $\Sigma_{w, 0}$ by $\Sigma_{w}$. If $w=s_{\alpha_{1}} \ldots s_{\alpha_{n}}$, $\alpha_{i} \in \widehat{R}_{\text {sim }}$, as a reduced decomposition, then

$$
\Sigma_{w}=\Sigma_{s_{\alpha_{1}}} \times \Xi \Sigma_{s_{\alpha_{2}}} \times \Xi \ldots \times \Xi \Sigma_{s_{\alpha_{n}}} .
$$

Suppose that we know that all the $\Sigma_{s_{\alpha_{i}}}(\xi)$ are closed. Then $\Sigma_{w}(\xi)$ is obtained by taking the closed subset $\Sigma_{s_{\alpha_{1}}}(\xi)$, for any point $\xi_{1}$ of this subset, taking the closed subset $\Sigma_{s_{\alpha_{2}}}\left(\xi_{1}\right)$, and so on, so $\Sigma_{w}(\xi)$ will be closed.

So we reduce to the case $w=s_{\alpha}, \alpha \in \widehat{R}_{\text {sim }}$. In this case the statement reduces to one about the group $S L_{2}(k)$ and its homogeneous space

$$
\Xi_{0}=S L_{2}(k) / B^{0}, \quad B^{0}=\left(\begin{array}{cc}
\mathcal{O}_{k}^{*} & k \\
0 & \mathcal{O}_{k}^{*}
\end{array}\right) \cap S L_{2}(k) .
$$

We have to prove that $B^{0}$-orbits in $\Xi_{0}$ are closed. But $\Xi_{0}=\left(k^{2}-\{0\}\right) / \mathcal{O}_{k}^{*}$, and $B^{0}$-orbits not reducing to single points, are the images of straight lines in $k^{2}-\{0\}$, so they are closed. The lemma is proved.

Having established the lemma, we get the convergence of $\tau_{w, l}(f)$ at the level of functions, and an argument similar to the proof of Proposition 4.1.6 shows that in this way we get a morphism of pro-objects as claimed in our theorem. The rest of the claims of the theorem (about $\tau_{e, l}$ ) are clear.

(4.3) Proof of Theorem 3.3.5. We first prove that $\tau_{w, l}\left(\mathcal{F}_{0}(\Xi)\right) \subset \mathcal{F}_{w}(\Xi)$. For this, it is enough to show the following.

(4.3.1) Lemma. Let $\xi \in \Xi, b=p(\xi) \in \widehat{F}$. Let $\gamma \in \widehat{W}$ be such that $U_{w}(b) \subset$ $\bar{U}_{\gamma}\left(b_{0}\right)$. Take a continuous section s of the $L_{\text {aff-torsor }} p^{-1}\left(\bar{U}_{\gamma}\left(b_{0}\right)\right) \rightarrow \bar{U}_{\gamma}\left(b_{0}\right)$ (i.e., of the restriction of $\Xi)$ and write, for any $b^{\prime} \in U_{w}(b)$,

$$
j_{b b^{\prime}}(\xi)=s\left(b^{\prime}\right)+a\left(b^{\prime}\right)
$$

so that $a: U_{w}(b) \rightarrow L_{\mathrm{aff}}$ is a locally constant function. Then the image of a is contained in some affine translation of $w\left(L_{\text {aff }}^{+}\right)$. 
Proof. As with some of the previous lemmas, this statement reduces to the case when $w$ is a simple affine reflection, i.e., to a similar statement about the $\mathbf{Z}$-torsor $\left(k^{2}-\{0\}\right) / \mathcal{O}_{k}^{*} \rightarrow P^{1}(k)$, which is elementary and left to the reader.

To prove the rest of the assertions of the theorem, we identify, via the Mellin transform (A.7.7),

$$
\mathcal{F}_{0}(\Xi) \simeq \Gamma_{\text {reg }}\left(\check{T}_{\text {aff }}, V\right), \quad \mathcal{F}^{\text {rat }}(\Xi) \simeq \Gamma_{\text {rat }}\left(\check{T}_{\text {aff }}, V\right) .
$$

After that, we notice that the Hecke operator

$$
\tau_{w, 0}: \mathcal{F}_{0}(\Xi) \rightarrow \mathcal{F}_{w}(\Xi)
$$

is given by the same formula $(3.3 .2)=(4.1 .5)$ as the operator induced by the principal series intertwiner $M_{w}$ on regular sections:

$$
\Gamma_{\text {reg }}\left(M_{w}\right): \Gamma_{\text {reg }}\left(\check{T}_{\text {aff }}, V\right) \rightarrow \Gamma_{\text {rat }}\left(\check{T}_{\text {aff }}, W^{*} V\right)=\Gamma_{\text {rat }}\left(\check{T}_{\text {aff }}, V\right)
$$

We conclude that $\tau_{w, 0}\left(\mathcal{F}_{0}(\Xi)\right) \subset \mathcal{F}_{w}^{\text {rat }}(\Xi)$ and define the operator $\tau_{w, 0}^{\text {rat }}$ to be the operator induced by $M_{w}$ on rational sections. Then, we define $\tau_{w, l}^{\text {rat }}=\tau_{0, l} \circ \tau_{w, 0}^{\text {rat }}$, where $\tau_{0, l}$ is the operator of shift by $l$. All the statements of the theorem follow now from the properties of the Mellin transform and of the intertwiners $M_{w}$.

(4.4) Proof of Theorem 3.3.8. (a) Because we can identify $\tau_{w}^{\text {rat }}$ with the intertwiner $M_{w}$, an element of $H_{\text {rat }}\left(\Gamma, \Delta_{1}\right)$ can be viewed as a finite formal linear combination $\sum_{w \in \widehat{W}} f_{w}(t) M_{w}$ with $f_{w} \in \mathbf{C}\left(\check{T}_{\text {aff }}\right)$. Since $A_{w}$ is just a rational multiple of $M_{w}$, we can as well think that $H_{\text {rat }}\left(\Gamma, \Delta_{1}\right)$ consists of formal linear combinations $\sum f_{w}(t) A_{w}$. Because the $A_{w}$ form a representation of $\widehat{W}$ in $\mathcal{F}^{\text {rat }}(\Xi)=\Gamma_{\text {rat }}\left(\check{T}_{\text {aff }}, V\right)$ and $V_{\lambda} \rightarrow V_{w * \lambda}$, a natural algebra structure on $H_{\text {rat }}\left(\Gamma, \Delta_{1}\right)$ is obtained by identifying it with the twisted group algebra $\mathbf{C}\left(\check{T}_{\text {aff }}\right)\left[\widehat{W}_{*}\right.$ formed with respect to the twisted action of $W$ on $\check{T}_{\text {aff }}$. In other words, $\mathbf{C}\left(\check{T}_{\text {aff }}\right)[\widehat{W}]_{*}$ consists of formal finite sums

$$
\sum_{w \in \widehat{W}} f_{w}(t)[w], \quad[w] \cdot\left[w^{\prime}\right]=\left[w w^{\prime}\right], \quad[w] f=f_{*}^{w}[w], \quad f_{*}^{w}(t):=f\left(w^{-1} * t\right) .
$$

The identification $\mathbf{C}\left(\check{T}_{\text {aff }}\right)[\widehat{W}]_{*} \rightarrow H_{\text {rat }}\left(\Gamma, \Delta_{1}\right)$ is just $[w] \rightarrow A_{w}$. Proposition 4.1.6 impies that in this way the map $\tau$ from (3.3.7) becomes a homomorphism of algebras.

(b) In view of Definition 2.3.3, Theorem 2.2.3 and of the difference of the two actions of $\widehat{W}$ on $\check{T}_{\text {aff }}$ (the "straight" action defining $\mathbf{C}\left(\check{T}_{\text {aff }}\right)[\widehat{W}]$ and the action (4.1.3) defining $\left.\mathbf{C}\left(\check{T}_{\text {aff }}\right)[\widehat{W}]_{*}\right)$, it is enough to prove the following.

(4.4.1) Proposition. In order that $\phi=\sum_{w \in \widehat{W}} f_{w}(t) A_{w} \in H_{\text {rat }}\left(\Gamma, \Delta_{1}\right)$ belong to $H\left(\Gamma, \Delta_{1}\right)$, it is necessary and sufficient that the following hold:

(1) The only possible singularities of each $f_{w}$ are poles of order $\leq 1$ on $\check{T}_{\alpha, q^{-1}}^{\mathrm{aff}}, \alpha \in$ $\widehat{R}_{+}$, with

$$
\operatorname{Res}_{T_{\alpha, q^{-1}}^{\text {aaf }}}\left(f_{w}\right)+\operatorname{Res}_{\widetilde{T}_{\alpha, q^{-1}}^{\text {aff }}}\left(f_{s_{\alpha} w}\right)=0, \quad w \in \widehat{W}, \alpha \in \widehat{R}_{+} .
$$

(2) Each $f_{w}$ vanishes along each $\check{T}_{\alpha, 1}^{\text {aff }}, \alpha \in D(w)$.

Proof. Let us summarize the relevant properties of the $A_{w}$ which follow from their definition and from the properties of the $M_{w}$ (Proposition 4.1.6): 
(4.4.2). Each $A_{w}$ has a first order pole along each $\check{T}_{\alpha, 1}^{\text {aff }}, \alpha \in D(w)$, and no other singularities.

Note that $s_{\alpha} * \lambda=\lambda$ for $\lambda \in \check{T}_{\alpha, q^{-1}}^{\text {aff }}$, so if $\lambda$ is a general point of $T_{\alpha, q^{-1}}^{\text {aff }}$, then $A_{s_{\alpha}}: V_{\lambda} \rightarrow V_{\lambda}$ is a well-defined involution (in the category of pro-vector spaces).

(4.4.3) Lemma. In the above situation, $A_{s_{\alpha}}: V_{\lambda} \rightarrow V_{\lambda}$ is the identity.

Proof. If $\alpha$ is a simple affine root, the statement reduces to that about the intertwiner $A$ in the unramified principal series of $S L_{2}(k)$. Namely, that the action of $A$ in the space of half-measures on $P^{1}(k)$ (i.e., of sections of the standard square root of the bundle of measures) is the identity. This is well known as the corresponding representation is irreducible [GGP]. The case of a general $\alpha \in \widehat{R}_{+}$is obtained from this by conjugation: we take $w \in \widehat{W}$ such that $w(\alpha)=\beta \in \widehat{R}_{\text {sim }}$; then $A_{w}$ intertwines the $A_{s_{\alpha}}$-action on $V_{\lambda}, \lambda \in \check{T}_{\alpha, q^{-1}}^{\text {aff }}$, and the $A_{s_{\beta}}$-action on $V_{\mu}$, $\mu=w * \lambda \in \check{T}_{\beta, q^{-1}}^{\mathrm{aff}}$. Lemma 4.4.3 is proved.

We need another lemma to pinpoint the origin of the conditions (1) of Proposition 4.4.1. Let $E$ be a free $\mathbf{C}[[x]]$-module (possibly of infinite rank). We denote by $\bar{E}=E / x E$ the vector space which can be thought of as the fiber of $E$ (as a bundle) at $x=0$. If $E=\left(E_{i}\right)_{i \in I}$ is a strict pro-object in the category of free $\mathbf{C}[[x]]$-modules, we will denote by $\bar{E}$ the pro-vector space $\left(\bar{E}_{i}\right)_{i \in I}$. Now let $\mathbf{Z} / 2=\{1, s\}$ act on $\mathbf{C}[[x]]$ by $s^{*}(x)=-x$ and suppose that $E$ is a $\mathbf{Z} / 2$-equivariant (pro-) free $\mathbf{C}[[x]]$-module. In other words, we have a $\mathbf{C}$-linear involution $s: E \rightarrow E$ such that $s(x e)=-x s(e)$. Then $s$ induces an involution $\bar{s}: \bar{E} \rightarrow \bar{E}$ of (pro-) vector spaces.

(4.4.4) Lemma. Suppose, in the situation described above, that $\bar{s}=\mathrm{Id}$. Consider $\mathbf{C}((x))[\mathbf{Z} / 2]$, the twisted group algebra with coefficients in $\mathbf{C}((x))$. It acts naturally on $E \otimes \mathbf{C}((x))$. In order that the action of $f_{0}(x)+f_{1}(x)[s]$ preserve $E$, it is necessary and sufficient that the following hold:

(a) $\operatorname{ord}_{x}\left(f_{i}\right) \geq-1, \quad i=0,1$.

(b) $\operatorname{Res}\left(f_{0}\right)+\operatorname{Res}\left(f_{1}\right)=0$.

The proof is left to the reader.

We now return to the proof of Proposition 4.4.1. Assume that $\sum_{w} f_{w}(t) A_{w}$ preserves the regular sections of $V$. As each $A_{w}$ does indeed have a pole along each $\check{T}_{\alpha, 1}^{\text {aff }}, \alpha \in D(w)$, condition (2) follows. Since the $A_{w}$ have no other singularities, each $f_{w}(\lambda)$ should be regular at every $\lambda$ whose stabilizer in $\widehat{W}$ is trivial, i.e., $\lambda \notin$ $\bigcup_{\alpha \in \widehat{R}_{+}} \check{T}_{\alpha, q^{-1}}^{\text {aff }}$. Further, by taking a generic point, say, $\lambda_{0}$, of some $\check{T}_{\alpha, q^{-1}}^{\text {aff }}$, we have $\operatorname{Stab}\left(\lambda_{0}\right)=\left\{1, s_{\alpha}\right\}$. By taking a 1-dimensional formal transversal slice to $\check{T}_{\alpha, q^{-1}}^{\text {aff }}$ at $\lambda_{0}$, we see that the rest of the conditions required in (1) follow from Lemmas 4.4.4 and 4.4.3. The proof of sufficiency of (1) and (2) for $\sum f_{w}(t) A_{w}$ to preserve regular sections is similar.

This finishes the proof of Proposition 4.4.1 and Theorem 3.3.8.

\section{Appendix. Analysis on ind/Pro objects}

(A.1) Instances of elementary description. For a category $\mathcal{C}$ we note by $\operatorname{Ind}(\mathcal{C})$ and $\operatorname{Pro}(\mathcal{C})$ the categories of ind- and pro-objects in $\mathcal{C}$. Their objects are filtering inductive or projective systems $\left(X_{i}\right)_{i \in I}$ over $\mathcal{C}$, i.e., co- or contravariant functors $I \rightarrow \mathcal{C}$, where $I$ is a small filtering category. For background, see AGV], Exp. I, 
and $[\mathrm{AM}]$. Sometimes we will use the notations "lim" $X_{i}, " \underline{\lim }$ " $X_{i}$ for the ind/proobject $\left(X_{i}\right)$. By $\operatorname{Ind}^{\aleph_{0}}(\mathcal{C}), \operatorname{Pro}^{\aleph_{0}}(\mathcal{C})$ we will denote the full subcategories formed

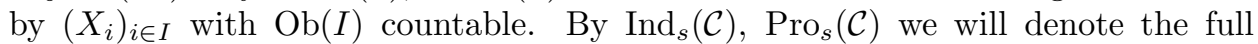
subcategories formed by strict ind- or pro-objects, i.e., by inductive (projective) systems in which all the structure maps are monomorphisms (epimorphisms).

We will need notation for the following categories (to be used in the main text): $\mathcal{S}_{0}, \mathcal{S}$ : finite sets, all sets.

Vect $_{0}$, Vect: finite-dimensional $\mathbf{C}$-vector spaces, all vector spaces.

$\operatorname{Mod}_{R}^{0}, \operatorname{Mod}_{R}(R$ a ring): finitely presented left $R$-modules, all left $R$-modules.

$\operatorname{Coh}(S), \mathrm{QCoh}(S)$ ( $S$ an algebraic variety over a field): coherent, quasi-coherent sheaves of $\mathcal{O}_{S}$-modules.

$\mathcal{P}$ : compact Hausdorff totally disconnected spaces.

$\mathcal{K} e$ : Kelley spaces [GZ], i.e., Hausdorff spaces $Z$ such that $U \subset Z$ is open iff $U \cap C$ is open in $C$ for any compact $C \subset Z$.

$\mathcal{K}$ : Kelley spaces which can be represented as a countable union of compact subspaces lying in $\mathcal{P}$.

The following is then elementary.

(A.1.1) Proposition. (a) The functors of taking the inductive or projective limit establish equivalences:

$$
\operatorname{Ind}\left(\mathcal{S}_{0}\right) \simeq \mathcal{S}, \quad \operatorname{Ind}\left(\operatorname{Mod}_{R}^{0}\right) \simeq \operatorname{Mod}_{R}, \quad \operatorname{Pro}\left(\mathcal{S}_{0}\right) \simeq \mathcal{P}, \quad \operatorname{Ind}_{s}^{\aleph_{0}}(\mathcal{P}) \simeq \mathcal{K} .
$$

(b) The functor $\varliminf_{\longleftarrow}: \mathrm{Pro}_{s}$ (Vect) $\rightarrow$ Vect is injective on Hom-sets.

In general, for an ind- or pro-object $X$ we will denote by $|X|$ the result of actually taking the limit of the inductive/projective system $X$. For example, if $X=\left(X_{i}\right)$ is a pro-object in $\mathcal{S}_{0}$, we will denote by $|X|=\varliminf_{i} X_{i}$ the corresponding object of $\mathcal{P}$, etc.

(A.2) Function spaces. For a finite set $X$ let $\mathcal{F}(X)$ be the space of functions $X \rightarrow$ C. This gives a contravariant functor $\mathcal{F}: \mathcal{S}_{0} \rightarrow$ Vect $_{0}$. We then extend it to pro-objects componentwise getting a functor, also denoted $\mathcal{F}$ :

$$
\mathcal{F}: \operatorname{Pro}\left(\mathcal{S}_{0}\right) \simeq \mathcal{P} \rightarrow \operatorname{Ind}\left(\text { Vect }_{0}\right) \simeq \operatorname{Vect} .
$$

For $Y \in \operatorname{Pro}\left(\mathcal{S}_{0}\right)$ the space $|\mathcal{F}(Y)|$ consists of locally constant functions $|Y| \rightarrow \mathbf{C}$, i.e., functions which are continuous if $\mathbf{C}$ is given the discrete topology. We then extend $\mathcal{F}$ to ind-objects componentwise, getting

$$
\mathcal{F}: \mathcal{K} \simeq \operatorname{Ind}_{s}^{\aleph_{0}}(\mathcal{P}) \rightarrow \operatorname{Pro}_{s}^{\aleph_{0}}(\text { Vect }) .
$$

Again, for $Z \in \operatorname{Ind}_{s}^{\aleph_{0}}(\mathcal{P})$ the space $|\mathcal{F}(Z)|$ consists of continuous functions $|Z| \rightarrow \mathbf{C}$ (with $\mathbf{C}$ discrete). But now the pro-object $\mathcal{F}(Z)$ contains more information than the vector space $|\mathcal{F}(Z)|$ (namely, the natural topology of the projective limit of discrete spaces). In the present paper the function space of a space from $\mathcal{K}$ will always be understood as a pro-object.

(A.3) 2-limits. We recall some general categorical constructions.

Let $\left(C_{i}\right)_{i \in I}$ be a filtering inductive system of categories, with structure functors denoted $C(\alpha): C_{i} \rightarrow C_{j}$ for $\alpha \in \operatorname{Hom}_{I}(i, j)$. The direct 2-limit $2 \mathfrak{l i m}_{i \in I} C_{i}$ is the category whose objects are pairs $\left(i, X_{i}\right)$ with $i \in I, X_{i} \in C_{i}$, with

$$
\operatorname{Hom}\left(\left(i, X_{i}\right),\left(j, X_{j}\right)\right)=\varliminf_{(k, \alpha, \beta) \in I /\{i, j\}} \operatorname{Hom}_{C_{k}}\left(C(\alpha)\left(X_{i}\right), C(\beta)\left(X_{j}\right)\right) .
$$


Here $I /\{i, j\}$ is the category whose objects are diagrams in $I$ of the form

$$
i \rightarrow k \leftarrow j
$$

with the obvious concept of morphisms. This category is a certain localization of the category called the quasi-colimit in Gra], p. 201, and the homotopy limit in Th].

If $\left(C_{i}\right)_{i \in I}$ is a filtering projective system of categories, with structure functors $C(\alpha): C_{j} \rightarrow C_{i}$ for $\alpha: i \rightarrow j$, then the inverse 2-limit $2 \varliminf_{i \in I} C_{i}$ is the category whose objects are systems $\left\{\left(E_{i}\right)_{i \in I},\left(\phi_{\alpha}\right)_{\alpha \in \operatorname{Mor}(I)}\right\}$ where $E_{i} \in C_{i}$ and $\phi_{\alpha}: C(\alpha)\left(E_{j}\right) \rightarrow E_{i}$ are isomorphisms, coimpactible in the obvious sense. A morphism in $2 \varliminf_{i} C_{i}$ from $\left\{\left(E_{i}\right),\left(\phi_{\alpha}\right)\right\}$ to $\left\{\left(E_{i}^{\prime}\right),\left(\phi_{\alpha}^{\prime}\right)\right\}$ is a family $\left(f_{i}: E_{i} \rightarrow E_{i}^{\prime}\right)$ compatible with the $\phi_{\alpha}, \phi_{\alpha}^{\prime}$. This construction is called a quasi-limit in [Gra], p. 217.

(A.4) Categories of bundles and torsors. Let $X$ be a finite set. A rank $r$ complex vector bundle on $X$ is just a collection $\left(V_{x}\right)_{x \in X}$ of $r$-dimensional Cvector spaces. Let $\operatorname{Bun}_{r}(X)$ be the category of such bundles. The correspondence $X \mapsto \operatorname{Bun}_{r}(X)$ gives a contravariant (2-)functor $\mathcal{S}_{0} \rightarrow \mathcal{C}$ at, and we extend it to $\operatorname{Ind}_{s}^{\aleph_{0}}\left(\operatorname{Pro}\left(\mathcal{S}_{0}\right)\right) \simeq \mathcal{K}$ by taking the 2-limits, i.e., setting

$$
\begin{aligned}
& \operatorname{Bun}_{r}\left(“ \varliminf^{\lim }{ }_{i \in I} Y_{i}\right)=2 \varliminf_{i \in I} \operatorname{Bun}_{r}\left(Y_{i}\right), \quad Y_{i} \in \mathcal{S}_{0}, \\
& \operatorname{Bun}_{r}\left(“ \varliminf^{\lim }{ }_{j \in J} Z_{j}\right)=2 \varliminf_{j \in J} \operatorname{Bun}_{r}\left(Z_{j}\right), \quad Z_{j} \in \operatorname{Pro}\left(\mathcal{S}_{0}\right) .
\end{aligned}
$$

Objects of these categories will be called topological $\mathbf{C}$-vector bundles. For a vector bundle $E$ on a finite set $X$ the space of sections $\Gamma(X, E)$ is an object of Vect ${ }_{0}$, for a bundle $E$ over a profinite set $Y$ the space $\Gamma(Y, E)$ is an object of $\operatorname{Ind}\left(\operatorname{Vect}_{0}\right)=$ Vect (i.e., just a vector space) and for a bundle over an object of $\mathcal{K}$ it is an object of $\mathrm{Pro}_{s}($ Vect). The following is elementary.

(A.4.3) Proposition. For $Y \in \operatorname{Pro}\left(\mathcal{S}_{0}\right)$ the category $\operatorname{Bun}_{r}(Y)$ is equivalent to the category of rank $r$ locally constant sheaves of $\mathbf{C}$-vector spaces on $|Y| \in \mathcal{P}$.

Such locally constant sheaves are the same as rank $r$ vector bundles given by locally constant transition functions.

The same approach will be followed in all other situations. For example, if $k$ is a local field such as in subsection (1.2), then for $Y \in \mathcal{P}$ we have the category $k-\operatorname{Bun}_{r}(Y)$ of topological $k$-vector bundles on $Y$ of rank $r$ (given by continuous, not necessarily locally constant, transition functions) and their continuous linear morphisms. The category of topological $k$-vector bundles on $Z=\left(Z_{i}\right)_{i \in I} \in \operatorname{Ind}_{s}^{\aleph_{0}}(\mathcal{P})$ is defined by

$$
k-\operatorname{Bun}_{r}(Z)=2 \varliminf_{i \in I} k-\operatorname{Bun}_{r}\left(Z_{i}\right) .
$$

Let $A$ be a free Abelian group of finite rank. For a finite set $X$ let $A$-Tors $(X)$ be the category of $A$-torsors over $X$. We extend the (2-)functor $A$-Tors : $\mathcal{S}_{0} \rightarrow \mathcal{C}$ at to $\operatorname{Ind}_{s}^{\aleph_{0}}\left(\operatorname{Pro}\left(\mathcal{S}_{0}\right)\right)$ as before:

$$
\begin{aligned}
& \left.A \text {-Tors ( "lim }{ }_{i \in I} Y_{i}\right)=2 \underline{\lim }_{i \in I} A \text {-Tors }\left(Y_{i}\right), \quad Y_{i} \in \mathcal{S}_{0}, \\
& \left.A \text {-Tors( " } \varliminf^{\lim }{ }_{j \in J} Z_{i}\right)=2 \varliminf_{j \in J} A \text {-Tors }\left(Z_{j}\right), \quad Z_{j} \in \operatorname{Pro}\left(\mathcal{S}_{0}\right) .
\end{aligned}
$$


(A.4.7) Proposition. Let $Y=" \varliminf{ }_{i \in I} Y_{i} \in \operatorname{Pro}\left(\mathcal{S}_{0}\right)$. For $B=\left(i, B_{i}\right) \in A$ $\operatorname{Tors}(Y)$ set

$$
|B|=\varliminf_{(\alpha, j) \in I / i} Y(\alpha)^{*} B_{i},
$$

where $I / i$ is the category of arrows $i \stackrel{\alpha}{\rightarrow} j$. Then $|B| \rightarrow|Y|$ is an A-torsor in a topological sense. The correspondence $B \mapsto|B|$ identifies $A$-Tors $(Y)$ with the category of such topological torsors.

(A.5) Function spaces on torsors. Let $A$ be as before, let $T_{A}=\operatorname{Spec} \mathbf{C}[A]$, and let $B$ be a principal homogeneous $A$-space. We denote by $\mathcal{F}(B)$, resp. $\mathcal{F}_{0}(B)$, the space of functions, resp. finitely supported functions, $B \rightarrow \mathbf{C}$. They are modules over $\mathbf{C}[A]$, and $\mathcal{F}_{0}(B)$ is free of rank 1. Let $\mathbf{C}\left(T_{A}\right)$ be the field of rational functions on $T_{A}$. Define

$$
\mathcal{F}^{\mathrm{rat}}(B)=\mathcal{F}_{0}(B) \otimes_{\mathbf{C}[A]} \mathbf{C}\left(T_{A}\right) .
$$

Let $\Lambda \subset A \otimes \mathbf{R}$ be a strictly convex cone. Denote by $\mathcal{F}_{\Lambda}(B) \subset \mathcal{F}(B)$ the space of functions whose support is contained in an affine translation of $\Lambda$. Clearly, $\mathcal{F}_{\Lambda}(A)$ is a ring (by convolution) containing $\mathcal{F}_{0}(A)=\mathbf{C}[A]$ and $\mathcal{F}_{\Lambda}(B)$ is an $\mathcal{F}_{\Lambda}(A)$-module.

An element $f: A \rightarrow \mathbf{C}$ of $\mathcal{F}_{\Lambda}(A)$ can be seen as a formal power series $\sum_{a \in A} f(a) t^{a}$. Let $\mathcal{F}_{\Lambda}^{\text {rat }}(A)$ be the set of $f$ for which this series is an expansion of a rational function. The conditions of $f$ imposed by saying that $f \in \mathcal{F}_{\Lambda}^{\text {rat }}(A)$ are translationally invariant, so for an $A$-torsor $B$ it makes sense to say that $f \in \mathcal{F}_{\Lambda}(B)$ is rational. So we get an $\mathcal{F}_{\Lambda}^{\text {rat }}(A)$-submodule $\mathcal{F}_{\Lambda}^{\text {rat }}(B) \subset \mathcal{F}_{\Lambda}(B)$. Note that we have a natural embedding (summation map)

$$
\Sigma_{\Lambda}: \mathcal{F}_{\Lambda}^{\mathrm{rat}}(B) \hookrightarrow \mathcal{F}^{\mathrm{rat}}(B) .
$$

Now let $X$ be a finite set and $B$ an $A$-torsor over $X$. The above constructions, applied pointwise (in $X$ ), give the spaces

$$
\begin{gathered}
\mathcal{F}_{0}(B) \in \operatorname{Mod}_{\mathbf{C}[A]}^{0}, \quad \mathcal{F}(B) \in \operatorname{Mod}_{\mathbf{C}[A]}, \quad \mathcal{F}^{\mathrm{rat}}(B) \in \operatorname{Mod}_{\mathbf{C}\left(T_{A}\right)}^{0}, \\
\mathcal{F}_{\Lambda}(B) \in \operatorname{Mod}_{\mathcal{F}_{\Lambda}(A)}^{0}, \quad \mathcal{F}_{\Lambda}^{\mathrm{rat}}(B) \in \operatorname{Mod}_{\mathcal{F}_{\Lambda}^{\text {rat }}(A)}^{0} .
\end{gathered}
$$

We then extend these constructions to torsors over objects of $\mathcal{K}=\operatorname{Ind}_{s}^{\aleph_{0}}\left(\operatorname{Pro}\left(\mathcal{S}_{0}\right)\right)$. For $Y=\left(Y_{i}\right)_{i \in I} \in \operatorname{Pro}\left(\mathcal{S}_{0}\right)$ and $B=\left(i, B_{i}\right) \in A$-Tors $(Y)$ set

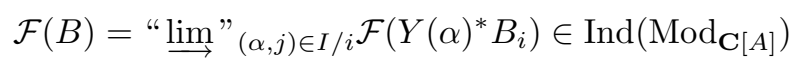

and similarly for $\mathcal{F}_{0}, \mathcal{F}^{\text {rat }}$, etc. Note that the vector space $|\mathcal{F}(B)|$ is contained in, but not, in general, equal to, the space of locally constant functions on the locally compact space $|B|$. On the other hand, $\operatorname{Ind}\left(\operatorname{Mod}_{\mathbf{C}[A]}^{0}\right)=\operatorname{Mod}_{\mathbf{C}[A]}$ so the object $\mathcal{F}_{0}(B)$ of the former category can be identified with the limit module $\left|\mathcal{F}_{0}(B)\right|$, and this module coincides with the space with compactly supported locally constant functions on $|B|$. Invoking (A.1.1)(a), we will thus consider $\mathcal{F}_{0}(B), \mathcal{F}^{\text {rat }}(B)$, $\mathcal{F}_{\Lambda}(B), \mathcal{F}_{\Lambda}^{\text {rat }}(B)$ simply as modules over their respective rings; more precisely, $\mathcal{F}_{0}(B)$ as a $\mathbf{C}[A]$-module, $\mathcal{F}^{\text {rat }}(B)$ as a $\mathbf{C}\left(T_{A}\right)$-vector space, $\mathcal{F}_{\Lambda}(B)$ as an $\mathcal{F}_{\Lambda}(A)$-module and $\mathcal{F}_{\Lambda}^{\text {rat }}(B)$ as an $\mathcal{F}_{\Lambda}^{\text {rat }}(A)$-module.

Next, let $Z=\left(Z_{j}\right)_{j \in J} \in \operatorname{Ind}_{s}^{\aleph_{0}}\left(\operatorname{Pro}\left(\mathcal{S}_{0}\right)\right)$ and $B \in A$-Tors $(Z)$, so $B$ is a system of torsors $B_{i}$ over $Z_{i}$ equipped with identifications $Z(\alpha)^{*} B_{j} \rightarrow B_{i}, \alpha: i \rightarrow j$. We 
define the function spaces on $B$ to be the pro-objects

$$
\begin{aligned}
\mathcal{F}_{0}(B) & =" \varliminf_{\lim } "{ }_{i \in I} \mathcal{F}_{0}\left(B_{i}\right) \in \operatorname{Pro}\left(\operatorname{Mod}_{\mathbf{C}[A]}\right), \\
\mathcal{F}(B) & =" \varliminf_{i \in I} \mathcal{F}\left(B_{i}\right) \in \operatorname{Pro}\left(\operatorname{Ind}\left(\operatorname{Mod}_{\mathbf{C}[A]}\right)\right),
\end{aligned}
$$

and so on.

(A.6) Rational maps of pro-vector bundles. Let $S$ be an irreducible affine algebraic variety over $\mathbf{C}$. We denote by $\Gamma_{\text {reg }}: \operatorname{QCoh}(S) \rightarrow \operatorname{Mod}_{\mathbf{C}[S]}$ the standard equivalence (the functor of regular sections) and by $\Gamma_{\text {rat }}: \operatorname{QCoh}(S) \rightarrow \operatorname{Mod}_{\mathbf{C}(S)}$ the functor of rational sections (i.e., the composition of $\Gamma_{\text {reg }}$ with the extension of scalars to $\mathbf{C}(S)$. For $\mathcal{F}, \mathcal{G} \in \mathrm{QCoh}(S)$ define

$$
\operatorname{Hom}_{\text {rat }}(\mathcal{F}, \mathcal{G})=\operatorname{Hom}_{\mathbf{C}(S)}\left(\Gamma_{\text {rat }}(\mathcal{F}), \Gamma_{\text {rat }}(\mathcal{G})\right) .
$$

Suppose $\mathcal{F}, \mathcal{G}$ are free (possibly of infinite rank). Then $R \in \operatorname{Hom}_{\text {rat }}(\mathcal{F}, \mathcal{G})$ can be given by a (possibly infinite) matrix $\left\|r_{i j}\right\|$ over $\mathbf{C}(S)$. For a rational function $a \in \mathbf{C}(S)$ we denote by $\operatorname{Sing}(a)$ its singular locus, i.e., the union of irreducible hypersurfaces $Z \subset S$ such that $\operatorname{ord}_{Z}(a)<0$. For a rational morphism $R: \mathcal{F} \rightarrow \mathcal{G}$ of free $\mathcal{O}_{S}$-modules and an irreducible hypersurface $Z \subset S$ we $\operatorname{define~}_{\operatorname{ord}_{Z}}(R)=$ $\inf _{i, j} \operatorname{ord}_{Z}\left(r_{i j}\right)$, where $\left\|r_{i j}\right\|$ is the matrix of $R$. This infimum (possibly equal to $(-\infty))$ is independent on the choice of bases. We set $\operatorname{Sing}(R)$ to be the union of $Z$ such that $\operatorname{ord}_{Z}(R)<0$.

Now let $\mathcal{F}=\left(\mathcal{F}_{i}\right), \mathcal{G}=\left(\mathcal{G}_{j}\right)$ be two strict pro-objects in the category of free $\mathcal{O}_{S}$-modules. According to the general definition of morphisms of pro-objects, we set

$$
\operatorname{Hom}_{\text {rat }}(\mathcal{F}, \mathcal{G})=\varliminf_{j} \varliminf_{i} \operatorname{lom}_{\text {rat }}\left(\mathcal{F}_{i}, \mathcal{G}_{j}\right) .
$$

So a rational morphism $A: \mathcal{F} \rightarrow \mathcal{G}$ is a compactible collection of rational morphisms $A^{(j)}: \mathcal{F}_{i(j)} \rightarrow \mathcal{G}_{j}$. We define

$$
\operatorname{Sing}(A)=\bigcup_{j} \operatorname{Sing}\left(A^{(j)}\right), \quad \operatorname{ord}_{Z}(A)=\inf _{j} \operatorname{ord}_{Z}\left(A^{(j)}\right) .
$$

It is clear that these concepts are unchanged under left or right composition with an isomorphism of pro-vector bundles.

(A.7) Mellin transform on torsors. Let $B$ be a principal homogeneous $A$-space. For $\lambda \in T_{A}$ let $\mathcal{L}(\lambda)_{B}$ denote the 1-dimensional space of functions $\psi: B \rightarrow \mathbf{C}$ which are homogeneous of degree $\lambda$, i.e., satisfy

$$
\psi(a+b)=\lambda^{a} \psi(b), \quad a \in A, b \in B .
$$

When $\lambda$ varies, the $\mathcal{L}(\lambda)_{B}$ form the fibers of an algebraic line bundle $\mathcal{L}_{B}$ on $T_{A}$. Let $B^{-}=\operatorname{Hom}_{A}(B, A)$ be the torsor dual to $B$, and let $i: T_{A} \rightarrow T_{A}$ be the inversion map: $i(\lambda)=\lambda^{-1}$. Notice that we have natural isomorphisms of algebraic line bundles

$$
\mathcal{L}_{B}^{*} \simeq \mathcal{L}_{B^{-}} \simeq i^{*} \mathcal{L}_{B}
$$

The following is obvious from the construction.

(A.7.2) Proposition. We have a natural identification

$$
m_{B}: \mathcal{F}_{0}(B) \rightarrow \Gamma_{\text {reg }}\left(T_{A}, \mathcal{L}_{B}^{*}\right) \simeq \Gamma_{\text {reg }}\left(T_{A}, i^{*} \mathcal{L}_{B}\right)=\Gamma_{\text {reg }}\left(T_{A}, \mathcal{L}_{B}\right) .
$$

It is defined as follows. For $\phi \in \mathcal{F}_{0}(B)$ the linear form $m_{B}(\phi): \mathcal{O}_{B} \rightarrow \mathcal{O}_{T_{A}}$ takes $\phi \in \mathcal{L}(\lambda)_{B}$ into $\sum_{b \in B} \phi(b) \psi(b)$. 
We will call $m_{B}$ the Mellin transform for $B$. Note that $m_{B}$ induces the identification

$$
m_{B}^{\text {rat }}: \mathcal{F}^{\text {rat }}(B) \rightarrow \Gamma_{\text {rat }}\left(T_{A}, \mathcal{L}_{B}\right) .
$$

If $X$ is a finite set of, say, $n$ elements, and $B$ is an $A$-torsor over $X$, then the above considerations can be applied to each fiber $B_{x}, x \in X$, and give, for any $\lambda \in T_{A}$, a line bundle $\mathcal{L}(\lambda) \in \operatorname{Bun}_{1}(X)$. Note that we have natural isomorphisms

$$
\mathcal{L}(\lambda) \otimes \mathcal{L}\left(\lambda^{\prime}\right) \simeq \mathcal{L}\left(\lambda \lambda^{\prime}\right)
$$

The space $\Gamma\left(X, \mathcal{L}(\lambda)\right.$ ) (of dimension $n$ ) will be denoted $V_{\lambda}$. When $\lambda$ varies, the $V_{\lambda}$ form the fibers of an algebraic vector bundle $V$ on $T_{A}$ of rank $n$, and we have natural identifications

$$
m_{B}: \mathcal{F}_{0}(B) \rightarrow \Gamma_{\text {reg }}\left(T_{A}, i^{*} V\right)=\Gamma_{\text {reg }}\left(T_{A}, V\right), \quad m_{B}^{\text {rat }}: \mathcal{F}^{\text {rat }}(B) \rightarrow \Gamma_{\text {rat }}\left(T_{A}, V\right) .
$$

Let $Y=\left(Y_{i}\right)_{i \in I} \in \operatorname{Pro}\left(\mathcal{S}_{0}\right)$ and $B=\left(i, B_{i}\right)$ be an $A$-torsor over $B$. We get line bundles $\mathcal{L}(\lambda)$ on $Y$ in the sense of (A.4.1), which satisfy (A.7.3). The space $V_{\lambda}=\Gamma(Y, \mathcal{L}(\lambda))$ is an object of $\operatorname{Ind}\left(\right.$ Vect $\left._{0}\right)$, so we can consider it as just a vector space $\left|V_{\lambda}\right|$. The "bundle" $V$ is an object of $\operatorname{Ind}\left(\operatorname{Coh}\left(T_{A}\right)\right)$, so we can identify it with the quasi-coherent sheaf $|V| \in \mathrm{QCoh}\left(T_{A}\right)$. The functor

$$
\Gamma_{\text {reg }}: \operatorname{Ind}\left(\operatorname{Coh}\left(T_{A}\right)\right) \rightarrow \operatorname{Ind}\left(\operatorname{Mod}_{\mathbf{C}[A]}^{0}\right)
$$

obtained by extending $\Gamma_{\text {reg }}: \operatorname{Coh}\left(T_{A}\right) \rightarrow \operatorname{Mod}_{\mathbf{C}[A]}^{0}$ to ind-objects is identified with the functor of global sections $\mathrm{QCoh}\left(T_{A}\right) \rightarrow \operatorname{Mod}_{\mathbf{C}[A]}$ so we have a Mellin transform $m_{B}$ as in (A.7.4), with $\Gamma_{\text {reg }}\left(T_{A}, V\right)$ understood in either of two senses. In addition, taking a trivialization of $B_{i}$ over $Y_{i}$, we get the following.

(A.7.5) Proposition. For $Y$ as above, the sheaf $|V|$ is free so it can be regarded as an algebraic vector bundle (possibly of infinite rank). The fiber of this bundle at $\lambda \in T_{A}$ is canonically identified with the vector space $\left|V_{\lambda}\right|$.

Next, we extend the above construction to an $A$-torsor $B$ over $Z=\left(Z_{i}\right)_{i \in I}$ from $\operatorname{Ind}_{s}^{\aleph_{0}}\left(\operatorname{Pro}\left(\mathcal{S}_{0}\right)\right)$. We get line bundles $\mathcal{L}(\lambda)$ on $Z$ in the sense of (A.4.2), which satisfy (A.7.3). The "spaces" $V_{\lambda}=\Gamma(Z, \mathcal{L}(\lambda))$ are objects of Pro(Vect). The "bundle" $V$ is an object of $\operatorname{Pro}\left(\mathrm{QCoh}\left(T_{A}\right)\right)$. The functors

$$
\begin{aligned}
& \Gamma_{\text {reg }}: \operatorname{Pro}\left(\operatorname{QCoh}\left(T_{A}\right)\right) \rightarrow \operatorname{Pro}\left(\operatorname{Mod}_{\mathbf{C}[A]}\right), \\
& \Gamma_{\text {rat }}: \operatorname{Pro}\left(\operatorname{QCoh}\left(T_{A}\right)\right) \rightarrow \operatorname{Pro}\left(\operatorname{Mod}_{\mathbf{C}\left(T_{A}\right)}\right)
\end{aligned}
$$

are defined componentwise. The Mellin transforms are now isomorphisms

$$
m_{B}: \mathcal{F}_{0}(B) \rightarrow \Gamma_{\text {reg }}\left(T_{A}, i^{*} V\right)=\Gamma_{\text {reg }}(A, V), \quad m_{B}^{\text {rat }}: \mathcal{F}^{\text {rat }}(B) \rightarrow \Gamma_{\text {rat }}\left(T_{A}, V\right)
$$

in $\operatorname{Pro}\left(\operatorname{Mod}_{\mathbf{C}[A]}\right)$. Unlike the previous case, neither of the pro-categories involved can be given an "elementary" description, so dealing with pro-objects is unavoidable at this stage.

\section{REFERENCES}

[AGV] M. Artin, A. Grothendieck, J.-L. Verdier, SGA4, Theorie des Topos et Cohomologie Etale des Schemas, t.1, Lecture Notes in Math. 269, Springer-Verlag, 1972. [MR 50:7130]

[AM] M. Artin, B. Mazur, Etale Homotopy, Lecture Notes in Math. 100, Springer-Verlag, 1969. MR 88a:14024

[B] K. Brown, Buildings, Springer-Verlag, 1989. MR 90e:20001 
[Cas] W. Casselman, Unramified principal series for p-adic groups I. The spherical function, Compositio Math. 40 (1980), 387-406. MR 83a:22018

[Ch] I. Cherednik, Double affine Hecke algebras and Macdonald's conjectures, Ann. Math. 141 (1995), 191-216. MR 96m:33010

[CK] N. Chriss, K. Khuri-Makdisi, On the Iwahori-Hecke algebra of a p-adic group, Int. Math. Res. Notices, 1998, no.2, 85-100. MR 99c:22023

[Dr] V.G. Drinfeld, Two-dimensional $l$-adic representations of the fundamental group of a curve over a finite field and automorphic forms on GL(2), Amer. J. Math. 105 (1983), 85-114. MR 86i:11066

[FP] T. Fimmel, A.N. Parshin, Introduction to Higher Adelic Theory, book in preparation.

[GZ] P. Gabriel, M. Zisman, Calculus of Fractions and Homotopy Theory (Ergebnisse der Math. 35), Springer-Verlag, 1967. MR 35:1019

[GG] H. Garland, I. Grojnowski, Affine Hecke algebras associated to Kac-Moody groups, preprint q-alg/9508019.

[GGP] I.M. Gelfand, M.I. Graev, I.I. Piatetski-Shapiro, Representation theory and automorphic functions, Academic Press, 1969. MR 38:2093

[GKV] V. Ginzburg, M. Kapranov, E. Vasserot, Residue construction of Hecke algebras, Adv. in Math. 128 (1997), 1-19. MR 98k:20074

[Gra] J.W. Gray, Formal Category Theory, Lecture Notes in Math. 391, Springer-Verlag, 1974. MR 51:8207

[Kac] V. Kac, Infinite-dimensional Lie algebras, Cambridge Univ. Press, 1985. MR 87e:17023

[Kat] K. Kato, The existence theorem for higher local class field theory, preprint M/80/43, IHES, 1980

[KL] D. Kazhdan, G. Lusztig, Proof of the Deligne-Langlands conjecture for Hecke algebras, Invent. Math. 87 (1987), 153-215. MR 88d:11121

[Lu1] G. Lusztig, Singularities, character formula and $q$-analog of weight multiplicity, Asterisque 101-102 (1983), 208-222. MR 85m:17005

[Lu2] G. Lusztig, Intersection cohomology methods in representation theory, Proc. ICM-90, vol.1, pp. 155-174, Math. Soc. Japan, Tokyo, 1991. MR 92m:20034

[Mat] H.Matsumoto, Sur les sous-groupes arithmétiques des groupes semi-simples déployés, Ann. Sci. ENS 2 (1969), 1-62. MR 39:1566

[Mil] J. Milnor, Introduction to Algebraic K-theory, Princeton Univ. Press, 1971. MR 50:2304

[Pa1] A.N. Parshin, On the arithmetic of 2-dimensional schemes. I, Repartitions and residues, Russian Math. Izv. 40 (1976), 736-773.

[Pa2] A.N. Parshin, Vector bundles and arithmetic groups I: The higher Bruhat-Tits tree, Proc. Steklov Inst. Math. 208 (1995), 212-233, preprint alg-geom/9605001. MR 2000j:20090

[PS] A. Pressley, G. Segal, Loop Groups, Clarendon Press, Oxford, 1986. MR 88i:22049

[Th] R.W. Thomason, Homotopy colimits in the category of small categories, Math. Proc. Cambridge Phil. Soc. 85 (1979), 91-109. MR 80b:18015

Department of Mathematics, University of Toronto, 100 St. George St., Toronto, Ontario, Canada M5S 3G3

E-mail address: kapranov@math.toronto.edu 